\title{
Approximate identities in Banach function algebras
}

by

\author{
H. G. DAles (Lancaster) and A. ÜLger (Istanbul)
}

\begin{abstract}
In this paper, we shall study contractive and pointwise contractive Banach function algebras, in which each maximal modular ideal has a contractive or pointwise contractive approximate identity, respectively, and we shall seek to characterize these algebras. We shall give many examples, including uniform algebras, that distinguish between contractive and pointwise contractive Banach function algebras. We shall describe a contractive Banach function algebra which is not equivalent to a uniform algebra. We shall also obtain results about Banach sequence algebras and Banach function algebras that are ideals in their second duals.
\end{abstract}

1. Introduction. Let $A$ be a Banach function algebra. A contractive approximate identity for $A$ is a bounded approximate identity of bound 1 ; $A$ is contractive if $A$ and all its maximal modular ideals have a contractive approximate identity. The uniform algebra $C_{0}(K)$ on a locally compact space $K$ is an example of a contractive Banach function algebra. The first main question that we shall address in this paper is how to characterize contractive Banach function algebras. Are there any examples other than those of the form $C_{0}(K)$ ? We shall prove that a natural uniform algebra is contractive if and only if it is a Cole algebra, so that every point of its character space is a $p$-point; in a future paper [13], we shall discuss the (wide) class of Banach function algebras that 'have a BSE norm' and prove more generally that every contractive Banach function algebra in this class is necessarily equivalent to a Cole algebra. However, we shall show in Example 5.2 that there are contractive Banach function algebras whose norm is not equivalent to the uniform norm.

A net $\left(f_{\alpha}\right)$ in a Banach function algebra $(A,\|\cdot\|)$ is a contractive pointwise approximate identity if $\left\|f_{\alpha}\right\| \leq 1$ for all $\alpha$ and $\lim _{\alpha} \varphi\left(f_{\alpha}\right)=1$ for each character $\varphi$ on $A ; A$ is pointwise contractive if $A$ and all its maximal modular ideals have contractive pointwise approximate identities, so that every con-

2010 Mathematics Subject Classification: Primary 46B15; Secondary 46B28, 46B42, 47L10. Key words and phrases: Banach function algebra, approximate identity, uniform algebra, Banach sequence algebra, Fourier algebra. 
tractive Banach function algebra is pointwise contractive. The second main question that we shall address is whether there are Banach function algebras in various classes that are pointwise contractive, but not contractive. We shall give several examples that distinguish between these two properties. For example, in Example 5.1, we shall describe a pointwise contractive Banach function algebra without any approximate identity, and, in Examples 4.8. (vi) and (viii), we shall give a pointwise contractive uniform algebra without a bounded approximate identity and a uniform algebra with a contractive pointwise approximate identity, but no approximate identity. We shall prove that a uniform algebra is pointwise contractive if and only if each point of its character space is a one-point Gleason part; this characterization will be extended to Banach function algebras with a BSE norm in [13].

We now summarize the main results of this paper.

In $\S 2$, we shall recall some notation and preliminary results about Banach function algebras. Our examples include the Fourier, Fourier-Stieltjes, and Figà-Talamanca-Herz algebras on locally compact groups, and various Segal algebras defined with respect to the Fourier algebra. In $\S 3$ we shall give a series of examples illustrating and distinguishing between various notions of approximate identity. Also we shall show in Theorem 3.5 that a natural, pointwise contractive Banach sequence algebra on a set $S$ is equivalent to $c_{0}(S)$. In Example 3.15 , we shall show that the minimum bound of a bounded pointwise approximate identity in a maximal modular ideal of a Fourier algebra $A(\Gamma)$ is 2 whenever $\Gamma$ is amenable as a discrete group. In $\S 4$, we shall prove that a uniform algebra on a locally compact space $K$ is contractive if and only if it is a Cole algebra, as defined in the text, and that it is pointwise contractive if and only if each Gleason part in $K$ is a singleton; we shall give many examples of uniform algebras delineating several properties.

In $\S 5$, we shall construct two unital Banach function algebras on closed intervals. The first gives a unital, pointwise contractive Ditkin algebra such that one of its maximal ideals does not have a bounded approximate identity. The second exhibits a unital, contractive Banach function algebra that is not equivalent to a uniform algebra.

2. Preliminaries. In this section we shall recall some notation, definitions, and standard results about Banach function algebras.

2.1. Banach function algebras. We set: $\mathbb{I}=[0,1]$, the closed unit interval; $\mathbb{D}=\{z \in \mathbb{C}:|z|<1\}$, the open unit disc in the plane; and $\mathbb{T}=\{z \in \mathbb{C}:|z|=1\}$, the unit circle. For $n \in \mathbb{N}$, we set $\mathbb{N}_{n}=\{1, \ldots, n\}$.

Let $E$ be a linear space, always taken to be over the complex field $\mathbb{C}$. Then $\operatorname{lin} F$ denotes the linear span of a subset $F$ of $E$; the set of extreme 
points of a convex set $S$ in $E$ is denoted by ex $S$; the convex hull of a subset $S$ of $E$ is denoted by $\langle S\rangle$.

Let $E$ be a Banach space. Then we denote the dual space of $E$ by $E^{\prime}$, with the duality specified by $(x, \lambda) \mapsto\langle x, \lambda\rangle, E \times E^{\prime} \rightarrow \mathbb{C}$; the second dual space of $E$ is $E^{\prime \prime}$. The closed ball of radius $r \geq 0$ in $E$ is $E_{[r]}$, and the weak-* topology on $E^{\prime}$ is $\sigma=\sigma\left(E^{\prime}, E\right)$.

Let $A$ be a (complex, associative) algebra. The set of characters on $A$ is denoted by $\Phi_{A}$. We set

$$
A^{[2]}=\{a b: a, b \in A\} \quad \text { and } \quad A^{2}=\operatorname{lin} A^{[2]} ;
$$

the algebra $A$ factors if $A=A^{[2]}$.

Let $A$ be an algebra that is also a Banach space with respect to a norm $\|\cdot\|$. Then $A$ is a Banach algebra if $\|a b\| \leq\|a\|\|b\|(a, b \in A)$; in the case where $A$ has an identity $e_{A}$, we also require that $\left\|e_{A}\right\|=1$. The Banach algebra of all bounded linear operators on a Banach space $E$ is denoted by $\mathcal{B}(E)$. For a monograph on Banach algebras, see [6]; we shall usually follow the notation of this book.

Let $(A,\|\cdot\|)$ be a Banach algebra. Then each $\varphi \in \Phi_{A}$ is a continuous linear functional on $A$ with $\|\varphi\| \leq 1$, and so we may regard $\Phi_{A}$ as a subset of $A_{[1]}^{\prime}$. The space $\Phi_{A}$ is locally compact with respect to the weak-* topology; it is compact in the case where $A$ has an identity, and is non-empty when $A$ is commutative and has an identity. In the case where $A$ is unital, we define

$$
K_{A}=\left\{\lambda \in A^{\prime}:\|\lambda\|=\left\langle e_{A}, \lambda\right\rangle=1\right\},
$$

the state space of $A$. The space $K_{A}$ is a non-empty, convex, and weak-* compact subset of $A_{[1]}^{\prime}$, and hence ${\overline{\left\langle\operatorname{ex~} K_{A}\right\rangle}}^{\sigma}=K_{A}$ by the Krĕn-Mil'man theorem. Clearly $\Phi_{A} \subset K_{A}$.

Let $A$ be a Banach algebra. The spaces $A^{\prime}$ and $A^{\prime \prime}$ are Banach $A$ bimodules, as in [6]. We denote the module operations on $A^{\prime}$ and $A^{\prime \prime}$ by $\cdot$, and set

$$
A \cdot A^{\prime}=\left\{a \cdot \lambda: a \in A, \lambda \in A^{\prime}\right\} \quad \text { and } \quad A A^{\prime}=\operatorname{lin} A \cdot A^{\prime} .
$$

There are two Arens products, denoted by $\square$ and $\diamond$, respectively, on the second dual space $A^{\prime \prime}$, each extending the module operations on $A^{\prime \prime}$; now $\left(A^{\prime \prime}, \square\right)$ and $\left(A^{\prime \prime}, \diamond\right)$ are Banach algebras each containing $A$ as a closed subalgebra. The algebra $A$ is Arens regular if $\mathrm{M} \square \mathrm{N}=\mathrm{M} \diamond \mathrm{N}\left(\mathrm{M}, \mathrm{N} \in A^{\prime \prime}\right)$. An element $\lambda \in A^{\prime}$ is weakly almost periodic if the map

$$
R_{\lambda}: a \mapsto a \cdot \lambda, \quad A \rightarrow A^{\prime},
$$

is weakly compact; the Banach space of all weakly almost periodic functionals on $A$ is denoted by $W A P(A)$. For details of the above, see [6, §2.6], and also [10, 11, 30]. 
The function constantly equal to 1 on a non-empty set $S$ is written as $1_{S}$ or just 1 .

Let $K$ be a non-empty, locally compact (Hausdorff) space. We write $C_{0}(K)$ and $C^{b}(K)$ for the spaces of all complex-valued, continuous functions on $K$ which vanish at infinity and which are bounded on $K$, respectively; we write $C(K)$ for $C_{0}(K)$ when $K$ is compact. Then $C^{b}(K)$ is a unital algebra with respect to the pointwise algebraic operations; the function $1_{K}$ is the identity of $C^{b}(K)$. We define

$$
|f|_{K}=\sup \{|f(x)|: x \in K\} \quad\left(f \in C^{b}(K)\right),
$$

so that $|\cdot|_{K}$ is the uniform norm on $K$ and $\left(C^{b}(K),|\cdot|_{K}\right)$ is a commutative, unital Banach algebra; $C_{0}(K)$ is a closed ideal in $C^{b}(K)$.

The support of a function $f \in C_{0}(K)$ is denoted by $\operatorname{supp} f$.

Let $K$ be a non-empty, locally compact space. A function algebra on $K$ is a subalgebra $A$ of $C^{b}(K)$ that separates strongly the points of $K$, in the sense that, for each $x, y \in K$ with $x \neq y$, there exists $f \in A$ with $f(x) \neq f(y)$, and, for each $x \in K$, there exists $f \in A$ with $f(x) \neq 0$. A function algebra $A$ is self-adjoint if $\bar{f} \in A$ whenever $f \in A$; here $\bar{f}(x)=\overline{f(x)}(x \in K)$. A Banach function algebra on $K$ is a function algebra $A$ on $K$ with a norm $\|\cdot\|$ such that $(A,\|\cdot\|)$ is a Banach algebra; a Banach function algebra $(A,\|\cdot\|)$ is equivalent to a uniform algebra if $\|\cdot\|$ and $|\cdot|_{K}$ are equivalent norms on $A$, so that $A$ is closed in $\left(C^{b}(K),|\cdot|_{K}\right) ;(A,\|\cdot\|)$ is a uniform algebra if $\|\cdot\|=|\cdot|_{K}$.

Let $A$ be a function algebra on $K$. For each $x \in K$, define

$$
\varepsilon_{x}(f)=f(x) \quad(f \in A) .
$$

Then each $\varepsilon_{x}$ is a character on $A$, called the evaluation character at $x$, and so we may regard $K$ as a subset of $\Phi_{A}$ by identifying $x \in K$ with $\varepsilon_{x} \in \Phi_{A}$. A Banach function algebra $A$ on $K$ is natural if $K=\Phi_{A}$ with this identification, and then $A \subset C_{0}(K)$ and $\|f\| \geq|f|_{K}(f \in A)$; clearly, $C_{0}(K)$ is natural and the uniform closure of $A$ in $C_{0}(K)$ is natural whenever $A$ is natural.

Let $(A,\|\cdot\|)$ be a natural Banach function algebra on a non-empty, compact space $K$. It follows from Šilov's idempotent theorem [6, Corollary 2.4.35] that $1_{K} \in A$, and so $A$ is unital; our definition requires that $\left\|1_{K}\right\|=1$.

Let $A$ be a commutative Banach algebra. For $\varphi \in \Phi_{A}$, set $M_{\varphi}=\operatorname{ker} \varphi$. Then $M_{\varphi}$ is a maximal modular ideal in $A$, and each maximal modular ideal has this form. In the case where $\Phi_{A} \neq \emptyset$, define $\widehat{a} \in C_{0}\left(\Phi_{A}\right)$ for $a \in A$ by $\widehat{a}(\varphi)=\varphi(a)\left(\varphi \in \Phi_{A}\right)$. Then the Gel'fand transformation

$$
\mathcal{G}: a \mapsto \widehat{a}, \quad A \rightarrow C_{0}\left(\Phi_{A}\right),
$$

is a norm-decreasing homomorphism from $A$ onto a function algebra $\widehat{A}: \widehat{A}$ is a natural Banach function algebra on $\Phi_{A}$ with respect to the quotient norm from $A$. The map $\mathcal{G}$ is injective whenever $A$ is semi-simple, and so we can 
regard each commutative, semi-simple Banach algebra as a natural Banach function algebra on $\Phi_{A}$.

Let $A$ be a Banach function algebra on a locally compact space $K$, and take $x \in K$. We write $M_{x}$ for $M_{\varepsilon_{x}}$ and set $M_{\infty}=A$. More generally, for a closed subset $E$ of $K$, we set

$$
I(E)=\{f \in A: f \mid E=0\},
$$

so that $I(E)$ is a closed ideal in $A$. Further, we write $J_{\infty}$ or $J_{\infty}(A)$ for the set of functions in $A$ of compact support, so that $J_{\infty}$ is an ideal in $A$, and we set

$$
J_{x}=J_{x}(A)=\left\{f \in J_{\infty}: x \notin \operatorname{supp} f\right\} \quad(x \in K),
$$

so that each $J_{x}$ is also an ideal in $A$ with $J_{x} \subset M_{x}$. We write $A_{0}$ for the closure of $J_{\infty}$ in $A$; the algebra $A$ is Tauberian if $A_{0}=A$, and so $A_{0}$ is a Tauberian Banach function algebra whenever $J_{\infty}$ separates strongly the points of $K$. A natural Banach function algebra $A$ is strongly regular if $J_{x}$ is dense in $M_{x}$ for each $x \in K \cup\{\infty\}$, and $A$ is regular if, for each non-empty, closed subset $F$ of $K$ and each $x \in K \backslash F$, there exists $f \in A$ with $f(x)=1$ and $f(y)=0(y \in F)$. A strongly regular algebra is regular. The algebra $A$ is a Ditkin algebra if $f \in \overline{f J_{x}}\left(f \in M_{x}\right)$ for each $x \in K \cup\{\infty\}$, so that a Ditkin algebra is strongly regular.

Let $A$ be a natural Banach function algebra on a locally compact, noncompact space $K$, and take $K_{\infty}=K \cup\{\infty\}$ to be the compact space that is the one-point compactification of $K$. We regard $A$ as a subalgebra of $C\left(K_{\infty}\right)$ by taking $f \in A$ to have the value 0 at the point $\infty$; the constant function on $K_{\infty}$ is 1 , and the unitization of $A$ is defined to be the subalgebra $A^{\sharp}=\{f+z 1: f \in A, z \in \mathbb{C}\}$ of $C\left(K_{\infty}\right)$. We define a norm $\|\cdot\|$ on $A^{\sharp}$ by setting

$$
\|f+z 1\|=\|f\|+|z| \quad\left(f+z 1 \in A^{\sharp}\right) .
$$

It is clear that $\left(A^{\sharp},\|\cdot\|\right)$ is a natural Banach function algebra on $K_{\infty}$ and that $A^{\sharp}$ contains $A$ as a maximal ideal. Further, $A^{\sharp}$ is equivalent to a uniform algebra on $K_{\infty}$ if and only if $A$ is equivalent to a uniform algebra on $K$.

For further details of the above theory, see [6], especially $\S \S 2.5,4.1$.

2.2. Approximate identities and units. Let $A$ be a Banach algebra. A net $\left(e_{\alpha}\right)$ in $A$ is an approximate identity for $A$ if

$$
\lim _{\alpha} a e_{\alpha}=\lim _{\alpha} e_{\alpha} a=a \quad(a \in A) ;
$$

an approximate identity is sequential if it is a sequence in $A$ indexed by $\mathbb{N}$; an approximate identity $\left(e_{\alpha}\right)$ is bounded if $\sup _{\alpha}\left\|e_{\alpha}\right\|<\infty$, and then $\sup _{\alpha}\left\|e_{\alpha}\right\|$ is the bound of the approximate identity; an approximate identity is contractive if it has bound 1 . We refer to a BAI and a CAI, respectively, in these two cases. For the theory of approximate identities in Banach algebras, see [6, §2.9]. 
Definition 2.1. Let $\left(A,\|\cdot\|_{A}\right)$ be a natural Banach function algebra on a non-empty, locally compact space $K$. A Banach function algebra $\left(B,\|\cdot\|_{B}\right)$ is an abstract Segal algebra (with respect to $A$ ) if $B$ is an ideal in $A$ and there is a net in $B$ that is an approximate identity for both $\left(A,\|\cdot\|_{A}\right)$ and $\left(B,\|\cdot\|_{B}\right)$.

Thus, in the above situation, $B$ is dense in $A, B$ is natural, and we may suppose that

$$
\|f\|_{A} \leq\|f\|_{B} \quad(f \in B) \quad \text { and } \quad\|f g\|_{B} \leq\|f\|_{A}\|g\|_{B} \quad(f \in A, g \in B) .
$$

A natural Banach function algebra $A$ on a non-empty, locally compact space $K$ is a strong Ditkin algebra if $M_{x}$ has a BAI contained in $J_{x}$ for each $x \in K \cup\{\infty\}$; see [6, Definition 4.1.31]. Clearly, a strong Ditkin algebra is a Ditkin algebra.

A commutative Banach algebra $(A,\|\cdot\|)$ has bounded approximate units of bound $m$ if, for each $a \in A$ and $\varepsilon>0$, there exists $u \in A_{[m]}$ with $\|a-u a\|<\varepsilon$. By [6, Proposition 2.9.14(ii)], $A$ has bounded approximate units of bound $m$ if and only if $A$ has a BAI of bound $m$. Suppose that $A$ has bounded approximate units of bound $m+\varepsilon$ for each $\varepsilon>0$. Then $A$ has bounded approximate units of bound $m$.

Let $A$ be a Banach function algebra on a non-empty, locally compact space $K$. Then $A$ has bounded relative approximate units (BRAUs) of bound $m$ if, for each non-empty, compact subset $L$ of $\Phi_{A}$ and each $\varepsilon>0$, there exists $f \in A_{[m]}$ with $|1-f(y)|<\varepsilon(y \in L)$. It is easy to see that $A$ has BRAUs of bound $m$ whenever $A$ has a BAI of bound $m$; Example 2.9, to be given below, will show that the converse need not hold.

TheOrem 2.2. Let $A$ be a Banach algebra with a BAI. Then:

(i) $A=A^{[2]}$ and $\overline{A A^{\prime}}=A \cdot A^{\prime}$;

(ii) $W A P(A) \subset A \cdot A^{\prime}$;

(iii) in the case where $A$ is Arens regular, $A^{\prime}=A \cdot A^{\prime}$.

Proof. (i) This is a form of Cohen's factorization theorem; considerably stronger forms of this theorem are given in [6, Theorem 2.9.24].

(ii) This is part of [9, Proposition 3.12].

(iii) By a result of [33] (also contained in [11]), $W A P(A)=A^{\prime}$ if and only if $A$ is Arens regular, and so this follows from (ii).

Definition 2.3. Let $A$ be a natural Banach function algebra on a nonempty, locally compact space $K$. Then $A$ is contractive if $M_{x}$ has a CAI for each $x \in K \cup\{\infty\}$.

We see quickly that uniform algebras of the form $C_{0}(K)$ are contractive, but it is not immediately obvious that there are any other contractive Banach function algebras; examples will be given in $\S 4$ and $\S 5$. 
Let $\left(A,\|\cdot\|_{A}\right)$ and $\left(B,\|\cdot\|_{B}\right)$ be natural Banach function algebras on a non-empty, locally compact space, and suppose that $A$ is a dense subalgebra of $B$ and that $\|f\|_{B} \leq\|f\|_{A}(f \in A)$. Then $B$ is contractive whenever $A$ is contractive.

Next we recall two classical Banach function algebras, and note that they are not contractive.

Example 2.4. As in [6, Example 2.1.13(ii)], we denote by $A(\overline{\mathbb{D}})$ the disc algebra of all functions $f$ in $C(\overline{\mathbb{D}})$ such that $f \mid \mathbb{D}$ is analytic. Thus $A(\overline{\mathbb{D}})$ is a natural uniform algebra on $\overline{\mathbb{D}}$. The maximal ideal $M_{z}$ at $z \in \overline{\mathbb{D}}$ has a CAI if $z \in \mathbb{T}$, and so $M_{z}=M_{z}^{[2]}$, but

$$
M_{z}^{[2]}=\overline{M_{z}^{2}}=\left\{f \in M_{z}: f^{\prime}(z)=0\right\} \subsetneq M_{z}
$$

when $z \in \mathbb{D}$, and so $M_{z}$ has no approximate identity when $z \in \mathbb{D}$. Thus $A(\overline{\mathbb{D}})$ is not contractive.

Let $A$ be a uniform algebra on a compact space $K$, and take $f \in A_{[1]}$ and $F \in A(\overline{\mathbb{D}})_{[1]}$. Then we note that $F \circ f \in A_{[1]}$. In particular, for $a \in \mathbb{D}$, set $\psi_{a}(z)=(z-a) /(1-\bar{a} z)(z \in \overline{\mathbb{D}})$, so that $\psi_{a}$ is a Möbius transformation with $\psi_{a}(a)=0$. Then $\psi_{a} \in A(\overline{\mathbb{D}})_{[1]}$, and so $\psi_{a} \circ f \in A_{[1]}$.

In the following result and later we shall use some specific functions that are defined on $\mathbb{I}$.

Definition 2.5. Take $t \in \mathbb{I}$ and $n \in \mathbb{N}$, and define $g_{n}$ to be the restriction to $\mathbb{I}$ of the function which is 0 on the interval $[t-1 / n, t+1 / n]$, which is equal to 1 outside the interval $[t-2 / n, t+2 / n]$, and which is linear on $[t-2 / n, t-1 / n]$ and $[t+1 / n, t+2 / n]$.

Then $\left(g_{n}\right)$ is a sequence in $C(\mathbb{I})$, and each $g_{n}$ is zero on a neighbourhood of $t$.

Example 2.6. Consider the algebra $A=B V C(\mathbb{I})$ of continuous functions of bounded variation on $\mathbb{I}$; this algebra is discussed in 6 , Theorem 4.4.35]. Fix $\gamma>0$. Here we note that $A$ is a natural, unital Banach function algebra with respect to the norm $\|\cdot\|_{\gamma}$ defined by

$$
\|f\|_{\gamma}=|f|_{\mathbb{I}}+\gamma \operatorname{var}_{\mathbb{I}}(f) \quad(f \in B V C(\mathbb{I})),
$$

where $\operatorname{var}_{\mathbb{I}}(f)$ is the variation of $f$ over $\mathbb{I}$; the algebra $A$ is regular, but it is not a uniform algebra.

Take $t \in \mathbb{I}$. Clearly the above sequence $\left(g_{n}\right)$ is contained in $J_{t}(A)$ and is a BAI of bound $1+2 \gamma$ for $M_{t}(A)$, and so $\left(B V C(\mathbb{I}),\|\cdot\|_{\gamma}\right)$ is a strong Ditkin algebra. However, this algebra is not contractive.

This example shows that we cannot replace 'CAI in each maximal ideal' by 'BAI of bound $c$ in each maximal ideal' for any $c>1$ when seeking contractive Banach function algebras. 
2.3. Banach sequence algebras. Let $S$ be a non-empty set. The algebra of all functions on $S$ of finite support is denoted by $c_{00}(S)$; the characteristic function of the singleton $\{s\}$ for $s \in S$ is denoted by $\delta_{s}$, so that $\delta_{s} \in c_{00}(S)(s \in S)$. The following definition is given in [6, §4.1].

Definition 2.7. Let $S$ be a non-empty set. A Banach sequence algebra on $S$ is a Banach function algebra $A$ on $S$ such that $c_{00}(S) \subset A$.

Let $A$ be a Banach sequence algebra on a set $S$. Then $J_{\infty}(A)=c_{00}(S)$ and $A_{0}$ is the closure of $c_{00}(S)$ in $A$. Thus a Banach sequence algebra is Tauberian if and only if $c_{00}(S)$ is dense in $A$. A natural Banach sequence algebra is always regular, and it is strongly regular if and only if it is Tauberian.

A form of converse to the following result will be given in Proposition 3.1 .

Proposition 2.8. Let $A$ be a Tauberian Banach sequence algebra on a non-empty set $S$. Then $A$ is natural and $A$ is an ideal in $A^{\prime \prime}$.

Proof. That $A$ is natural is [6, Proposition 4.1.35(i)]. To see that $A$ is an ideal in $A^{\prime \prime}$, set $R_{f}(g)=g f(g \in A)$ for each $f \in A$, so that $R_{f} \in \mathcal{B}(A)$. For each $f \in c_{00}(S)$, the operator $R_{f}$ has finite-dimensional range, and so is compact. Since $c_{00}(S)$ is dense in $A, R_{f}$ is compact, and hence weakly compact, for each $f \in A$. By [30, Proposition 1.4.13], $A$ is an ideal in $A^{\prime \prime}$.

ExAmple 2.9. For $\alpha=\left(\alpha_{k}\right) \in \mathbb{C}^{\mathbb{N}}$, set

$$
p_{n}(\alpha)=\frac{1}{n} \sum_{k=1}^{n} k\left|\alpha_{k+1}-\alpha_{k}\right| \quad(n \in \mathbb{N}), \quad p(\alpha)=\sup \left\{p_{n}(\alpha): n \in \mathbb{N}\right\},
$$

and define $A$ to be $\left\{\alpha \in c_{0}: p(\alpha)<\infty\right\}$, so that $A$ is a self-adjoint Banach sequence algebra on $\mathbb{N}$ for the norm given by

$$
\|\alpha\|=|\alpha|_{\mathbb{N}}+p(\alpha) \quad(\alpha \in A) .
$$

The details of this example, which is due to Feinstein, are given in 6 , Example 4.1.46]. It is shown that $A$ is natural, and that, for each $m \in \mathbb{N}$ and each compact subset $K$ of $\mathbb{N}$ with $m \notin K$, there exists $\alpha \in A$ with $\alpha(m)=0$, with $\alpha(j)=1(j \in K)$, and with $\|\alpha\| \leq 4$. Thus each maximal modular ideal of $A$ has BRAUs of bound 4. It is also shown that $A^{2}=A_{0}^{2}=A_{0}$, that $A_{0}$ is separable, and that $A$ is non-separable, and so $A^{2}$ is a closed subspace of infinite codimension in $A$. Thus $A$ is not Tauberian and $A$ does not have any approximate identity.

The following remarkable example of Blecher and Read from [2] was the first to exhibit a natural Banach sequence algebra that has a BAI, but which is not Tauberian. 
EXAMPLE 2.10. There is a natural Banach sequence algebra $A$ on $\mathbb{N}$ with all of the following properties:

(i) $A$ is self-adjoint, and so dense in $c_{0}$;

(ii) $A$ has a CAI, and so $A^{[2]}=A$;

(iii) there exists $g \in A$ such that the singly-generated subalgebra $\operatorname{lin}\left\{g^{n}: n \in \mathbb{N}\right\}$ is dense in $A$, and so $A$ is separable;

(iv) $A$ is non-Tauberian, and $A / A_{0}$ is an infinite-dimensional space;

(v) the closed ideal $A_{0}$ also has a CAI;

(vi) each maximal modular ideal in $A$ has a BAI (but there is no upper bound to the bounds of these BAIs);

(vii) the CAI for $A_{0}$ is contained in $c_{00}$, and so $A_{0}$ is a strong Ditkin algebra;

(viii) $A$ is Arens regular, but $A$ is not an ideal in $A^{\prime \prime}$.

2.4. Pointwise approximate identities. There is a related notion concerning approximate identities in Banach function algebras. The following definition originates with Jones and Lahr [27]; see also [26, 29, 37. However, our terminology is different.

Definition 2.11. Let $A$ be a natural Banach function algebra on a nonempty, locally compact space $K$. A net $\left(e_{\alpha}\right)$ in $A$ is a pointwise approximate identity (PAI) if

$$
\lim _{\alpha} e_{\alpha}(x)=1 \quad(x \in K) ;
$$

the PAI is bounded, with bound $m>0$, if $\sup _{\alpha}\left\|e_{\alpha}\right\| \leq m$, and then $\left(e_{\alpha}\right)$ is a bounded pointwise approximate identity (BPAI); a bounded pointwise approximate identity of bound 1 is a contractive pointwise approximate identity (CPAI). The algebra $A$ is pointwise contractive if $M_{x}$ has a CPAI for each $x \in K \cup\{\infty\}$.

Thus a natural Banach function algebra $A$ on $K$ is pointwise contractive if and only if, for each $x \in K \cup\{\infty\}$, each non-empty, finite subset $F$ of $K$ with $x \notin F$, and each $\varepsilon>0$, there exists $f \in M_{x}$ with $\|f\| \leq 1$ and $|1-f(y)|<\varepsilon(y \in F)$.

Clearly each Banach function algebra has a PAI. A Banach function algebra with BRAUs has a BPAI, with the same bound. Example 2.9 exhibited a Banach sequence algebra with a BPAI, but no approximate identity. In Examples 3.13 and 3.14 , it will be shown that there are natural Banach function algebras $A$ that have a CPAI, but no BAI. Example 4.8(vi) will give a natural, pointwise contractive uniform algebra that is not contractive, and Example 4.8(viii) will give a natural uniform algebra with a CPAI, but no approximate identity. In Example 5.1, we shall exhibit a natural, pointwise contractive Banach function algebra that does not have any approximate identity. 
As we stated at the beginning of this paper, our aim is to understand the structure of contractive and pointwise contractive Banach function algebras.

Proposition 2.12. Let $A$ be a natural, Banach function algebra on a non-empty, locally compact space $K$, and take $x \in K$. Suppose that there exists $n>0$ such that, for each neighbourhood $U$ of $x$, there exists $g \in A_{[n]}$ with $g(x)=1$ and $\operatorname{supp} g \subset U$.

(i) Suppose that $\overline{J_{x}}=M_{x}$ and $A$ has a BAI of bound $m$. Then $M_{x}$ has $a$ BAI of bound $m(1+n)$.

(ii) Suppose that $A$ has a BPAI of bound $m$. Then $M_{x}$ has a BPAI of bound $m(1+n)$.

Proof. (i) Take $h \in M_{x}$ and $\varepsilon>0$. Then there exists $h_{1} \in J_{x}$ with $\left\|h-h_{1}\right\|<\varepsilon$. Since $A$ has a BAI of bound $m$, there exists $f \in A_{[m]}$ with $\left\|h_{1}-f h_{1}\right\|<\varepsilon$. There is a neighbourhood $U$ of $x$ with $U \cap \operatorname{supp} h_{1}=\emptyset$, and so, by hypothesis, there exists $g \in A_{[n]}$ with $g(x)=1$ and $\operatorname{supp} g \subset U$, so that $h_{1} g=0$. Now we have $f-f g \in\left(M_{x}\right)_{[m(n+1)]}$ and

$$
\begin{aligned}
\|h-h(f-f g)\| & \leq\left\|h-h_{1}\right\|+\left\|h_{1}-f h_{1}\right\|+\left\|h-h_{1}\right\|\|f-f g\| \\
& <\varepsilon+\varepsilon+\varepsilon m(1+n) .
\end{aligned}
$$

Thus $M_{x}$ has bounded approximate units of bound $m(n+1)$, and hence a BAI of bound $m(1+n)$.

(ii) Take a non-empty, finite subset $F$ of $K$ with $x \notin F$, and take $\varepsilon>0$. Then there exists $f \in A_{[m]}$ with $|1-f(y)|<\varepsilon(y \in F)$. Take a neighbourhood $U$ of $x$ with $U \cap F=\emptyset$, and then take $g \in A_{[n]}$ with $g(x)=1$ and $\operatorname{supp} g \subset U$. Set $h=f-f g \in\left(M_{x}\right)_{[m(n+1)]}$. We see immediately that $|1-h(y)|<\varepsilon(y \in F)$, and so $M_{x}$ has a BPAI of bound $m(1+n)$.

The next examples exhibit some Banach function algebras whose maximal ideals do not have a BPAI.

EXAMPLE 2.13. The maximal ideals of the natural Banach function algebras $C^{(n)}(\mathbb{I})($ for $n \in \mathbb{N})$ and $\left(\operatorname{Lip}_{\alpha}(\mathbb{I}),\|\cdot\|_{\alpha}\right)$ (for $\left.0<\alpha \leq 1\right)$, which are defined in [6, §4.4], do not have a BPAI. For example, for each $n \in \mathbb{N}$ and $f \in \operatorname{Lip}_{\alpha}(\mathbb{I})$ with $f(0)=0$ and $f(1 / n)>1 / 2$, necessarily $\|f\|_{\alpha} \geq n^{\alpha} / 2$.

Example 2.14. Let $A$ be the space $\ell^{p}=\ell^{p}(\mathbb{N})$, where $p \geq 1$. Then $A$ is a natural, self-adjoint, Tauberian Banach sequence algebra on $\mathbb{N}$, and so $A$ is an ideal in $A^{\prime \prime}$; in the case where $p>1, A$ is reflexive. Clearly $A$ and each maximal modular ideal in $A$ have approximate identities, but $A$ does not have a BPAI. Here $A^{[2]}=A^{2}=\ell^{p / 2}$.

ExAmple 2.15. Let $\omega: \mathbb{N} \rightarrow[1, \infty)$ be a function, and set

$$
B_{\omega}=\left\{\alpha \in c_{0}: p_{\omega}(\alpha):=\sum_{i=1}^{\infty} \omega(i)\left|\alpha_{i+1}-\alpha_{i}\right|<\infty\right\} ;
$$


for $\alpha \in B_{\omega}$, set $\|\alpha\|_{\omega}=|\alpha|_{\mathbb{N}}+p_{\omega}(\alpha)$, as in [12, p. 33]. Then $B_{\omega}$ is a natural, Tauberian Banach sequence algebra on $\mathbb{N}$. A slight extension of [12, Theorem 3.10.1] shows that the following are equivalent:

(a) $B_{\omega}$ has a BAI;

(b) $B_{\omega}$ has BRAUs;

(c) $B_{\omega}$ has a BPAI;

(d) $\liminf _{n \rightarrow \infty} \omega(n)<\infty$.

Various other equivalent properties involving amenability are given in the quoted theorem. Further remarks about this example will be given in [13].

2.5. Peaking properties. Let $A$ be a function algebra on a non-empty, locally compact space $K$. A closed subset $F$ of $K$ is a peak set if there exists a function $f \in A$ with $f(x)=1(x \in F)$ and $|f(y)|<1(y \in K \backslash F)$; in this case, $f$ peaks on $F$; a point $x \in K$ is a peak point if $\{x\}$ is a peak set, and a $p$-point if $\{x\}$ is an intersection of peak sets. The set of $p$-points of $A$ is denoted by $\Gamma_{0}(A)$; it is sometimes called the Choquet boundary of $A$. In the case where $A$ is a Banach function algebra, a countable intersection of peak sets is always a peak set, and so, when $K$ is metrizable, $\Gamma_{0}(A)$ is the set of peak points of $A$. (However, even a uniform algebra may have $p$-points which are not peak points.) A closed subset $L$ of $K$ is a closed boundary for $A$ if $|f|_{L}=|f|_{K}(f \in A)$; the intersection of all the closed boundaries for $A$ is called the Silov boundary, $\Gamma(A)$ [6, Definition 4.3.1(iv)]. Suppose that $K$ is compact and that $A$ is a natural uniform algebra on $K$. Then, by [6, Corollary 4.3.7(i)], $\Gamma(A)=\overline{\Gamma_{0}(A)}$ and $\Gamma(A)$ is a closed boundary. Suppose that $K$ is compact and metrizable and that $A$ is a natural Banach function algebra on $K$. Then, by [5] (see also [6, Corollary 4.3.7(ii)]), the set of peak points is dense in $\Gamma(A)$.

The following theorem is proved by a small modification of the proof of [6, Theorem 4.3.5].

TheOREM 2.16. Let $A$ be a Banach function algebra on a non-empty, compact space $K$, and take $x \in K$. Suppose that $M_{x}$ has BRAUs. Then $x$ is a $p$-point for $A$.

We now obtain a necessary condition for a Banach function algebra $A$ to be contractive.

TheOREM 2.17. Let $A$ be a natural Banach function algebra on a nonempty, locally compact space $K$ such that each maximal modular ideal of $A$ has a BAI. Then $\Gamma_{0}(A)=K$.

Proof. Take $x \in K$. Then $M_{x}$ has a BAI, and hence $M_{x}$ has BRAUs. By Theorem 2.16, $x$ is a $p$-point for $A$. 
Corollary 2.18. Let $A$ be a natural, contractive Banach function algebra on a non-empty, locally compact space $K$. Then $\Gamma_{0}(A)=K$.

We shall see in Example 4.8(vi) and Theorem 4.9 that we cannot replace 'contractive' by 'pointwise contractive' in the above corollary, even in the case where $A$ is a uniform algebra.

3. Relations among approximate identities. In this section we shall give some results and examples showing the relationships between various notions of approximate identity for Banach function algebras.

3.1. Examples. The first result, which is a (weak) converse to Proposition 2.8, is essentially given in [29, Theorem 3.1]; a similar result is contained in [2, Corollary 1.3].

Proposition 3.1. Let $A$ be a Banach function algebra such that $A$ is an ideal in $A^{\prime \prime}$ and $A$ has a BPAI. Then $A$ also has a BAI, with the same bound. In the case where the BPAI is contained in $A_{0}$, the algebra $A$ is Tauberian.

Proof. Let $\left(e_{\alpha}\right)$ be a BPAI for $A$ with bound $m$. Then $\left(e_{\alpha}\right)$ has a weak-* accumulation point in $\left(A^{\prime \prime}\right)_{[m]}$, and we may suppose, by passing to a subnet, that weak-*- $\lim _{\alpha} e_{\alpha}=e \in\left(A^{\prime \prime}\right)_{[m]}$. For each $f \in A$, we know that $e \cdot f$ belongs to $A$ because $A$ is an ideal in $A^{\prime \prime}$. Further, for each $\varphi \in \Phi_{A}$, we have

$$
\varphi(f)=\left\langle f, \varepsilon_{\varphi}\right\rangle=\lim _{\alpha}\left\langle e_{\alpha} f, \varepsilon_{\varphi}\right\rangle=\left\langle e, f \cdot \varepsilon_{\varphi}\right\rangle=\left\langle e \cdot f, \varepsilon_{\varphi}\right\rangle=\varphi(e \cdot f),
$$

and so $e \cdot f=f$. Thus $e_{\alpha} f \rightarrow f$ in $(A, \sigma)$, where $\sigma=\sigma\left(A, A^{\prime}\right)$. By [6. Proposition 2.9.14(iii)], $A$ has a BAI with bound $m$.

Now suppose that $\left(e_{\alpha}\right)$ is contained in $A_{0}$, and again take $f \in A$. Then the net $\left(e_{\alpha} f\right)$ is in $A_{0}$, and so $f \in{\overline{A_{0}}}^{\sigma}$. By Mazur's theorem, ${\overline{A_{0}}}^{\sigma}=A_{0}$, and so $f \in A_{0}$. Thus $A$ is Tauberian.

We shall show in Example 3.9 that a Banach sequence algebra $A$ can be an ideal in $A^{\prime \prime}$ without being Tauberian, but we do not know, even in the case of Banach sequence algebras, whether a Banach function algebra $A$ that is an ideal in $A^{\prime \prime}$ and has a BPAI is necessarily Tauberian.

Proposition 3.2. Let $A$ be a natural Banach function algebra on a non-empty, locally compact space $K$ such that $A$ is reflexive as a Banach space. Take $x \in K$, and suppose that $M_{x}$ has a BPAI. Then $M_{x}$ has an identity, $x$ is an isolated point of $K$, and $K$ is compact.

Proof. Since $A$ is reflexive, the closed balls of $M_{x}$ are weakly compact, and so the BPAI in $M_{x}$ has a weakly convergent subnet, with weak limit $f$, say. Clearly $f(y)=1(y \in K \backslash\{x\})$, and so $f$ is the identity of $M_{x}$. Since $f \in C_{0}(K)$, the point $x$ is an isolated point of $K$ and $K$ is compact. 
We now give examples of unital Banach function algebras which are reflexive as Banach spaces (and so are ideals in their second duals) and have connected character spaces, so that they are not Banach sequence algebras. Their maximal ideals do not have a BPAI. We are grateful to David Blecher for reminding us of these examples.

EXAMPle 3.3. Let $S$ be either $\mathbb{Z}$ or $\mathbb{Z}^{+}$. Then $\omega=\left(\omega_{n}: n \in S\right)$ is a weight sequence on $S$ if $\omega_{n}>0(n \in S), \omega_{0}=1$, and

$$
\omega_{m+n} \leq \omega_{m} \omega_{n} \quad(m, n \in S) .
$$

Fix $p>1$ and a weight sequence $\omega$ on $S$, and consider the Banach space

$$
\ell^{p}(S, \omega)=\left\{\alpha=\left(\alpha_{n}: n \in S\right):\|\alpha\|_{p, \omega}=\left(\sum_{n \in S}\left|\alpha_{n}\right|^{p} \omega_{n}^{p}\right)^{1 / p}<\infty\right\} .
$$

It is well-known that $\ell^{p}(S, \omega)$ is a Banach algebra for convolution multiplication, $\star$, provided that there exists a constant $C>0$ such that

$$
\sum_{m+n=k} \frac{\omega_{k}^{q}}{\omega_{m}^{q} \omega_{n}^{q}} \leq C \quad(k \in S),
$$

where $q$ is the conjugate index to $p$. This follows from Hölder's inequality; the first explicit reference that we have found is [23, Theorem 6.7(D)]. Hence $\alpha \star \beta \in \ell^{p}\left(\mathbb{Z}^{+}, \omega\right)$ with $\|\alpha \star \beta\|_{p, \omega} \leq C^{1 / q}\|\alpha\|_{p, \omega}\|\beta\|_{p, \omega}$. To obtain a Banach function algebra that satisfies our precise definition, we must replace the given norm by an equivalent one. The interesting fact about these examples is that, as Banach spaces, they are reflexive (this is not affected by the re-norming), and so certainly they are Arens regular and ideals in their second duals.

Now choose $\omega_{n}=(1+|n|)^{r}(n \in S)$, where $r>1 / q$. It is easy to see that $\omega=\left(\omega_{n}: n \in S\right)$ is a weight sequence on $S$ that satisfies the inequality (3.1), and so $\ell^{p}\left(\mathbb{Z}^{+}, \omega\right)$ and $\ell^{p}(\mathbb{Z}, \omega)$ are Banach algebras. Again by Hölder's inequality, $\ell^{p}(\mathbb{Z}, \omega) \subset \ell^{1}(\mathbb{Z})$. Both these algebras are semi-simple and they are natural, unital Banach function algebras on $\mathbb{T}$ and $\overline{\mathbb{D}}$, respectively. Let $M_{z}$ be a maximal ideal of either of these algebras. Since $z$ is not an isolated point of the character space of the algebra, it follows from Proposition 3.2 that this maximal ideal does not have a BPAI.

Proposition 3.4. Let $A$ be a pointwise contractive Banach function algebra on a non-empty, locally compact space $K$. Suppose that $F$ and $G$ are disjoint, non-empty, finite subsets of $K$, and take $\varepsilon>0$. Then there exists $f \in A_{[1]}$ such that $|1-f(x)|<\varepsilon(x \in F)$ and $f(x)=0(x \in G)$.

Proof. Set $k=|G|$, and choose $\eta \in(0, \varepsilon / k)$. For each $y \in G$, there exists $f_{y} \in A_{[1]}$ with $f_{y}(y)=0$ and $\left|1-f_{y}(x)\right|<\eta(x \in F)$. Now define

$$
f=\prod\left\{f_{y}: y \in G\right\} \text {. }
$$


Then clearly $f \in A_{[1]}$ and $f(y)=0(y \in G)$. For each $x \in F$, we have

$$
|1-f(x)| \leq \sum\left\{\left|1-f_{y}(x)\right|: y \in G\right\}<k \eta<\varepsilon,
$$

as required.

The following result shows that the only natural, pointwise contractive Banach sequence algebra on a set $S$ is $c_{0}(S)$.

TheOrem 3.5. Let $A$ be a natural Banach sequence algebra on a nonempty set $S$. Suppose that $A$ is pointwise contractive. Then $A$ is equivalent to the uniform algebra $c_{0}(S)$.

Proof. Take $F$ and $G$ to be disjoint, closed, non-empty subsets of $S_{\infty}$.

First, suppose that $F$ and $G$ are contained in $S$, and hence finite. By Proposition 3.4 there exists $f \in A_{[1]}$ with $|1-f(x)|<1 / 2(x \in F)$ and $f \mid G=0$. Second, suppose that $\infty \in G$. Then $F$ is finite. Since $A$ is pointwise contractive, there exists $f \in A_{[1]}$ with $|1-f(x)|<1 / 4(x \in F)$. Set

$$
H=\{y \in G:|f(y)| \geq 1 / 4\},
$$

so that $H$ is a compact, and hence finite, subset of $S$ and $F \cap H=\emptyset$. By Proposition 3.4, there exists $g \in A_{[1]}$ with $|1-g(x)|<1 / 2(x \in F)$ and $g \mid H=0$. Set $h=f g$, so that $h \in A_{[1]}$. Then $|1-h(x)|<1 / 2(x \in F)$ and $|h(y)|<1 / 4(y \in G)$. Thus the hypotheses of [6, Theorem 4.1.19] are satisfied (with $m=1$ ), and so, by that theorem, $A^{\sharp}$ is equivalent to $C\left(S_{\infty}\right)$.

It follows that $A$ is equivalent to $c_{0}(S)$.

ExAmple 3.6. There is a norm $\|\cdot\|$ on $c_{0}$ such that $\left(c_{0},\|\cdot\|\right)$ is a contractive Banach function algebra that is equivalent to a uniform algebra, but is not a uniform algebra.

Indeed, let $B$ be the closed unit ball of $\left(c_{0},|\cdot|_{\mathbb{N}}\right)$, and set

$$
C=\left\{\left(x_{n}\right) \in B:\left|x_{1}-x_{2}\right| \leq 1\right\} .
$$

Then it is easily checked that $C$ is absolutely convex and closed and that $(1 / 2) B \subset C \subset B$; further, $x y \in C$ whenever $x, y \in C$. Thus $C$ is the closed unit ball of a norm, say $\|\cdot\|$, on $c_{0}$ such that $\left(c_{0},\|\cdot\|\right)$ is a Banach function algebra that is equivalent to a uniform algebra; it is not a uniform algebra because $\|(-1,1,0,0,0, \ldots)\|=2$. Finally, we check that $\left(c_{0},\|\cdot\|\right)$ is contractive. For example, the sequence $\left(\sum_{j=2}^{n} \delta_{j}\right)$ is a CAI for the maximal modular ideal $\left\{\left(x_{n}\right) \in c_{0}: x_{1}=0\right\}$.

We now consider when the existence of a CPAI for a Banach function algebra $A$ implies the existence of a BAI or even just an approximate identity for $A$. We have already shown in Proposition 3.1 that, in the case where $A$ is an ideal in $A^{\prime \prime}$, the existence of a CPAI implies that of a CAI.

The first counter-example is the original one of Jones and Lahr [27]; it shows that a Banach function algebra can have a CPAI without having any 
approximate identity. We shall give further (some easier) related examples in Examples 3.13, 3.14, 3.17, and 5.1, we shall also give examples of pointwise contractive uniform algebras without any BAI in Example 4.8(vi) and Theorem 4.9 and an example of a uniform algebra with a CPAI, but no approximate identity, in Example 4.8(vii).

EXAmple 3.7. Let $S=\left(\mathbb{Q}^{+\bullet},+\right)$ be the semi-group of strictly positive rational numbers. The semi-group algebra $A=\left(\ell^{1}(S), \star,\|\cdot\|_{1}\right)$ is a commutative, semi-simple Banach algebra. Then it is shown in [27] that there is a strictly increasing sequence $\left(n_{d}\right)$ in $\mathbb{N}$ such that the sequence $\left(\delta_{1 / n_{d}}\right)$ is a CPAI for $A$. However, $A$ does not have any approximate identity. Indeed, for each $x \in S$, we have $\left\|\delta_{x}-\delta_{x} \star f\right\|_{1} \geq 1(f \in A)$.

Some future examples of natural Banach function algebras which are pointwise contractive, but which have no approximate identity, will be based on the following proposition.

Proposition 3.8. Let $(A,\|\cdot\|)$ be a natural Banach function algebra on a non-empty, locally compact space $K$. Suppose that $f_{0} \in C_{0}(K) \backslash A$ is such that $f f_{0} \in A$ and $\left\|f f_{0}\right\| \leq\|f\|$ for each $f \in A \cup\left\{f_{0}\right\}$. Set $B=A \oplus \mathbb{C} f_{0}$, with

$$
\left\|f+z f_{0}\right\|=\|f\|+|z| \quad(f \in A, z \in \mathbb{C}) .
$$

Then $B$ is a natural Banach function algebra on $K$ containing $A$ as a proper closed ideal. Further, $B^{2} \subset A$, and so $B$ does not have an approximate identity.

Suppose that $A$ has a CPAI or is pointwise contractive. Then $B$ has a CPAI or is pointwise contractive, respectively.

Proof. It is clear that $B$ is a Banach function algebra on $K$ and that $B$ contains $A$ as a proper closed ideal.

Take $\varphi \in \Phi_{B}$. Then $\varphi \mid A \in \Phi_{A}$, and so there exists $x \in K$ such that $\varphi(f)=f(x)(f \in A)$. Now take $f \in A$ such that $\varphi(f)=f(x)=1$. Then $\varphi\left(f_{0}\right)=\varphi\left(f f_{0}\right)=\left(f f_{0}\right)(x)=f_{0}(x)$, and hence $\varphi(g)=g(x)(g \in B)$. Thus $B$ is natural on $K$.

Clearly $B^{2} \subset A \subsetneq B$, and so $B$ does not have an approximate identity.

Let $M_{x}(A)$ be a maximal ideal of $A$. Then a CPAI in $M_{x}(A)$ is also a CPAI in $M_{x}(B)$, the corresponding maximal ideal of $B$.

The following example is a first concrete realization of the above proposition; others will be given in Examples 3.17 and 5.1 .

EXAMPLE 3.9. Set $A=\ell^{2}$ (with pointwise multiplication), take $f_{0}$ to be the sequence $(1 / \sqrt{n}: n \in \mathbb{N})$, and set $B=A \oplus \mathbb{C} f_{0}$, as in Proposition 3.8. Then the conditions of the proposition are satisfied, and so $B$ is a natural Banach sequence algebra on $\mathbb{N}$. Further, as a Banach space, $B$ is reflexive, 
and so certainly $B$ is an ideal in $B^{\prime \prime}$. However, $B$ is not Tauberian and $B$ does not have an approximate identity; indeed, $B_{0}=A \subsetneq B$. The algebra $B$ does not have a BPAI.

3.2. Banach function algebras on locally compact groups. Let $G$ be a locally compact group. The group algebra on $G$ is $L^{1}(G)=\left(L^{1}(G), \star\right)$ and the measure algebra is $M(G)=(M(G), \star)$, so that $M(G)$ is a unital Banach algebra, and $L^{1}(G)$ is a closed ideal in $M(G)$; the algebra $L^{1}(G)$ has a CAI. See [6, §3.3], for example.

Let $\Gamma$ be a locally compact group with identity $e_{\Gamma}$ and left Haar measure $m_{\Gamma}$. Then the Fourier algebra and Fourier-Stieltjes algebra on $\Gamma$ are denoted by $A(\Gamma)$ and $B(\Gamma)$, respectively; $B(\Gamma)$ is a Banach function algebra on $\Gamma$, and $A(\Gamma)$ is a closed ideal in $B(\Gamma)$. As a Banach space, $B(\Gamma)$ is the dual of the group $C^{*}$-algebra $C^{*}(\Gamma)$, and so we have a weak-* topology $\sigma\left(B(\Gamma), C^{*}(\Gamma)\right)$ on $B(\Gamma)$; for details, see [15]. In the case where $\Gamma$ is the dual group of a locally compact, abelian (LCA) group $G, A(T)$ and $B(\Gamma)$ are identified with the spaces of Fourier transforms of elements of $L^{1}(G)$ and Fourier-Stieltjes transforms of elements of $M(G)$, respectively.

The theory of Fourier and Fourier-Stieltjes algebras originates in the seminal work of Eymard [15].

More generally, take $p>1$. Then the Figà-Talamanca-Herz algebra, $A_{p}(\Gamma)$, is described in [6, pp. 493-494]; the Fourier algebra $A(\Gamma)$ is the algebra $A_{2}(\Gamma)$. By [6. Theorem 4.5.31], $A_{p}(\Gamma)$ is a self-adjoint, natural, strongly regular Banach function algebra on $\Gamma$, and $A_{p}(\Gamma)$ is dense in $\left(C_{0}(\Gamma),|\cdot|_{\Gamma}\right)$. The particular maximal modular ideal

$$
A_{e}(\Gamma)=\left\{f \in A(\Gamma): f\left(e_{\Gamma}\right)=0\right\}
$$

is the augmentation ideal of $A(\Gamma)$.

The following proposition combines results from [38] and [3, Corollary 2.8].

Proposition 3.10. Let $\Gamma$ be a locally compact group, take $p>1$, and set $A=A_{p}(\Gamma)$. Then $\Phi_{A}$ is $\sigma\left(A^{\prime}, A^{\prime \prime}\right)$-closed in $A^{\prime}$ if and only if $\Gamma$ is amenable.

Let $A$ be a natural Banach function algebra. Then it is immediate (see [38. Proposition 2.8]) that $\Phi_{A}$ is $\sigma\left(A^{\prime}, A^{\prime \prime}\right)$-closed in $A^{\prime}$ whenever $A$ has a BPAI. Hence we obtain the following result.

Proposition 3.11. Let $\Gamma$ be a locally compact group, and take $p>1$. Then the following are equivalent:
(a) $\Gamma$ is amenable;
(b) $A_{p}(\Gamma)$ has a BPAI;
(c) $A_{p}(\Gamma)$ has a $\mathrm{BAI}$;
(d) $A_{p}(\Gamma)$ has a CAI. 
Proof. The equivalence of (a), (c), and (d) is well-known; for example, see [6, Theorem 4.5.32]. Clearly (c) implies (b). Now suppose that $A=A_{p}(\Gamma)$ has a BPAI. Then $\Phi_{A}$ is $\sigma\left(A^{\prime}, A^{\prime \prime}\right)$-closed in $A^{\prime}$, and so $\Gamma$ is amenable by Proposition 3.10 .

COROLlary 3.12. Let $\Gamma$ be an amenable locally compact group, and take $p>1$ and $x \in \Gamma$. Then $M_{x}\left(A_{p}(\Gamma)\right)$ has a BAI of bound 2 .

Proof. We may suppose that $x=e_{\Gamma}$. By considering elements of the form $\left(\chi_{V} \star \chi_{V}\right) / m_{\Gamma}(V)$ in $A_{p}(\Gamma)$ for a suitable symmetric, compact neighbourhood $V$ of $e_{\Gamma}$, we see that, for each neighbourhood $U$ of $e_{\Gamma}$, there exists $g \in A_{p}(\Gamma)_{[1]}$ with $g\left(e_{\Gamma}\right)=1$ and $\operatorname{supp} g \subset U$. The result follows from Proposition 2.12(i) (with $m=n=1$ ), where we recall that $A_{p}(\Gamma)$ is strongly regular, and so $\overline{J_{x}\left(A_{p}(\Gamma)\right)}=M_{x}\left(A_{p}(\Gamma)\right)$.

Let $G$ be a LCA group. A subalgebra $S$ of $\left(L^{1}(G), \star\right)$ with a norm $\|\cdot\|_{S}$ is a Segal algebra on $G$ if $S$ is dense in $\left(L^{1}(G),\|\cdot\|_{1}\right)$, if $\left(S,\|\cdot\|_{S}\right)$ is a Banach algebra such that $\|f\|_{S} \geq\|f\|_{1}(f \in S)$, if $S$ is invariant under all the translations $S_{a}$ for $a \in G$, if $\left\|S_{a} f\right\|_{S}=\|f\|_{S}(f \in S, a \in G)$, and if the map

$$
a \mapsto S_{a} f, \quad G \rightarrow S,
$$

is continuous for each $f \in S$. These Segal algebras are abstract Segal algebras with respect to the Banach function algebra $L^{1}(G)$, in the sense of Definition $2.1[6, \S 4.5]$.

We now give two examples of natural Banach function algebras $A$ such that $A$ has a CPAI and each maximal modular ideal of $A$ has a BPAI, but $A$ has no BAI.

EXAMPLE 3.13. Let $G$ be a non-discrete LCA group with dual group $\Gamma$, and take $p \geq 1$. We define

$$
S_{p}(G)=\left\{f \in L^{1}(G): \widehat{f} \in L^{p}(\Gamma)\right\}
$$

and

$$
\|f\|_{S_{p}}=\max \left\{\|f\|_{1},\|\widehat{f}\|_{p}\right\} \quad\left(f \in S_{p}(G)\right) .
$$

Then $\left(S_{p}(G), \star\right)$ is a Segal algebra with respect to $L^{1}(G)$ that is identified with a natural Banach function algebra on $\Gamma$; see [6, Example 4.5.27(iii)], where the norm has a slightly different form. Then, by [6, Proposition 4.5.28], $S_{p}(G)^{2} \subsetneq S_{p}(G)$, and so, by Theorem 2.2(i), $S_{p}(G)$ does not have a BAI. Indeed, it is clear that $S_{p}(G)$ does not have BRAUs. However, by [25, Theorem 3.1], $S_{p}(G)$ has a CPAI whenever $G$ is also non-compact. (Note that our $S_{p}(G)$ is called ' $A_{p}(G)$ ' in [25].)

Thus, for example, $S_{p}(\mathbb{R})$ has a CPAI, but no BRAUs, for each $p \geq 1$.

Take $\gamma \in \Gamma$, say $\gamma=e_{\Gamma}$, and take a compact neighbourhood $U$ of $e_{\Gamma}$ with $m_{\Gamma}(U) \leq 1$. As in Corollary 3.12 , there exists $g \in A(\Gamma)$ with $g\left(e_{\Gamma}\right)=\|g\|=1$ 
and with supp $g \subset U$, say $g=\widehat{f}$, where $f \in L^{1}(G)$ and $\|f\|_{1}=1$. Since $|g|_{\Gamma} \leq 1$, we see that the norm of $g$ in $L^{p}(\Gamma)$ is at most $m_{\Gamma}(U)^{1 / p} \leq 1$, and so $\|f\|_{S_{p}} \leq 1$. By Proposition 2.12(ii), each maximal modular ideal in $S_{p}(G)$ has a BPAI of bound 2 .

EXAMPLE 3.14. Let $G$ be a non-compact LCA group with dual group $\Gamma$, and take $p>1$. We define

$I_{p}(G)=L^{1}(G) \cap L^{p}(G) \quad$ and $\quad\|f\|_{I_{p}(G)}=\max \left\{\|f\|_{1},\|f\|_{p}\right\} \quad\left(f \in I_{p}(G)\right)$.

Then $\left(I_{p}(G), \star\right)$ is a Segal algebra with respect to $L^{1}(G)$ that is identified with a natural Banach function algebra on $\Gamma$. By [25, Theorem 2.1], $I_{p}(G)$ has a CPAI. However, $I_{p}(G)$ does not have BRAUs and $I_{p}(G)$ does not factor.

Take a neighbourhood $U$ of $e_{\Gamma}$. As above, there exists $g \in A(\Gamma)$ with $g\left(e_{\Gamma}\right)=\|g\|=1$ and with $\operatorname{supp} g \subset U$, say $g=\widehat{f}$, where $f \in L^{1}(G)$ with $\|f\|_{1}=1$. Since $g \in L^{1}(\Gamma)$ and $f$ is the (inverse) Fourier transform of $g$, we see that $f \in C_{0}(G)$ with $|f|_{G} \leq 1$. Thus the norm of $f$ in $L^{p}(G)$ is bounded by

$$
\left(\int_{G}|f(x)|^{p-1}|f(x)| d m_{G}(x)\right)^{1 / p} \leq\left(\int_{G}|f(x)| d m_{G}(x)\right)^{1 / p}=\|f\|_{1}^{1 / p}=1 .
$$

This shows that $f \in I_{p}(G)_{[1]}$. Again, this implies that each maximal modular ideal in $I_{p}(G)$ has a BPAI of bound 2.

EXAMPLE 3.15. Let $\Gamma$ be an amenable locally compact group, and take $p>1$. We recall from Corollary 3.12 that each maximal modular ideal of $A_{p}(\Gamma)$ has a BAI of bound 2 .

Let $I$ be a closed ideal in $A(\Gamma)$. By combining [29, Theorem 5.3] and [19, Lemma 2.2], we see that the following are equivalent:

(a) I has a BPAI;

(b) $I$ has the form $I(H)$ for some $H$ in the closed coset ring of $\Gamma$;

(c) I has a BAI.

Take $H$ to be a closed subgroup of $\Gamma$. Then it is proved by Delaporte and Derighetti [14, Theorems 10 and 11] and by Kaniuth and Lau [28, Theorem 3.4] that 2 is the best bound for a BAI in $I(H)$ whenever $H$ is closed, normal, and non-open and when $H$ is open and $\Gamma / H$ is infinite. This applies when $H=\left\{e_{\Gamma}\right\}$ and $\Gamma$ is infinite, and so 2 is the minimum bound of a BAI for each maximal modular ideal of $A(\Gamma)$ whenever $\Gamma$ is infinite. For further results, see [20].

We next consider the best bound for a BPAI in a maximal ideal of $A(\Gamma)$ in the case where $\Gamma$ is infinite, and $\Gamma_{d}$, the discrete version of $\Gamma$, is amenable (which implies that $\Gamma$ is amenable). It is enough to consider the augmenta- 
tion ideal $A_{e}(\Gamma)$. By [15, 2.24], $B(\Gamma)$ embeds isometrically in $B\left(\Gamma_{d}\right)$; since $A(\Gamma) \subset B(\Gamma)$, we may suppose that $A(\Gamma)$ is a subalgebra of $B\left(\Gamma_{d}\right)$.

Take $\left(u_{\alpha}\right)$ to be a BPAI in $A_{e}(\Gamma)$ of bound $m$, and consider $\left(u_{\alpha}\right)$ as a net in $B\left(\Gamma_{d}\right)_{[m]}$. By passing to a subnet, we may suppose that $\left(u_{\alpha}\right)$ converges in the weak-* topology $\sigma\left(B\left(\Gamma_{d}\right), C^{*}\left(\Gamma_{d}\right)\right)$, say to $u \in B\left(\Gamma_{d}\right)_{[m]}$. Since the evaluation functionals are in $C^{*}\left(\Gamma_{d}\right)$, convergence in this weak-* topology implies pointwise convergence on $\Gamma$, and so $u(\gamma)=1\left(\gamma \in \Gamma \backslash\left\{e_{\Gamma}\right\}\right)$ and $u\left(e_{\Gamma}\right)=0$.

By Proposition 3.11. $A\left(\Gamma_{d}\right)$ has a CAI, say $\left(v_{\beta}\right)$. Then $\left(u v_{\beta}\right)$ is a net in $A_{e}\left(\Gamma_{d}\right)_{[m]}$ such that

$$
\lim _{\beta} f u v_{\beta}=\lim _{\beta} f v_{\beta}=f \quad\left(f \in A_{e}\left(\Gamma_{d}\right)\right),
$$

and so $\left(u v_{\beta}\right)$ is a BAI in $A_{e}\left(\Gamma_{d}\right)$ of bound $m$. By the above remark, $m \geq 2$ whenever $\Gamma_{d}$ is infinite and amenable, and so 2 is the minimum bound of a BPAI for each maximal modular ideal of $A(\Gamma)$ in this case.

In the case where $\Gamma$ is a locally compact group that is not amenable, $A(\Gamma)$ does not have a BPAI; if $\Gamma$ is amenable, but not amenable as a discrete group (for example, $\Gamma=S O(3)$ ), we do not know the best bound for a BPAI in $A(\Gamma)$.

As remarked in [20, Remark 2.5], it seems to be open whether the bound of 2 for a BAI in maximal modular ideal of $A_{p}(\Gamma)$ is optimal in the case where $p>1$ and $p \neq 2$.

There is a large and significant class of Banach function algebras known as BSE algebras [37. This class includes the Fourier algebra $A(\Gamma)$ of an amenable group $\Gamma$ [29], and the two Banach algebras $S_{p}(G)$ and $I_{p}(G)$ considered in Examples 3.13 and 3.14 [25]. As will be proved in [13], the multiplier algebra of a pointwise contractive BSE algebra on a locally compact space $K$ is isomorphic to $C^{b}(K)$. This shows that none of the algebras $A(\Gamma)$, $S_{p}(G)$, or $I_{p}(G)$ is pointwise contractive. In particular, as is the case here, the algebra $A$ is certainly not pointwise contractive when the multiplier algebra of a BSE algebra is weakly sequentially complete.

EXAMPLE 3.16. Let $\omega$ be a weight on an infinite LCA group $G$ (so that $\omega: G \rightarrow[1, \infty)$ is such that $\omega(s+t) \leq \omega(s) \omega(t)(s, t \in G)$ and $\left.\omega\left(e_{G}\right)=1\right)$. Since $L^{1}(G)$ is not pointwise contractive, the Beurling algebra $\left(L^{1}(G, \omega), \star\right)$ also fails to be pointwise contractive.

The following example, which shows that a Banach function algebra can have a CPAI without having any approximate identity, is easier than the one given in Example 3.7 .

EXAMPle 3.17. Take $\left(L^{1}(G), \star\right)$ for a non-discrete, LCA group $G$, and take a singular measure $\mu_{0} \in M(G)_{[1]} \backslash L^{1}(G)$ such that $\mu_{0} \star \mu_{0} \in L^{1}(G)$; a 
proof that such an element $\mu_{0}$ exists for each such group $G$ is given in [24]. Since $L^{1}(G)$ is a closed ideal in $M(G)$, it follows that $f \star \mu_{0} \in L^{1}(G)$ with $\left\|f \star \mu_{0}\right\|_{1} \leq\|f\|_{1}\left(f \in L^{1}(G)\right)$, and also $\left\|\mu_{0} \star \mu_{0}\right\|_{1} \leq\left\|\mu_{0}\right\|$. We regard $\mu_{0}$ as an element of $C_{0}(\Gamma)$, where $\Gamma$ is the dual group to $G$. Set $B=L^{1}(G) \oplus \mathbb{C} \mu_{0}$. Then $B$ satisfies the conditions given in Proposition 3.8 .

In this case, $L^{1}(G)$ has a CAI and each maximal ideal of $L^{1}(G)$ has a BAI of bound 2. Thus $B$ has a CPAI and each maximal ideal of $B$ has a BPAI of bound 2. Since maximal modular ideals of $L^{1}(G)$ have BRAUs with bound 2 , the same is true for $B$. However, $B$ does not have an approximate identity.

\section{Uniform algebras}

4.1. Cole algebras. First recall that every $C^{*}$-algebra $A$ is Arens regular and that $\left(A^{\prime \prime}, \square\right)$ is a $C^{*}$-algebra [6, Theorem 3.2.36]; in particular, $\left(C_{0}(K)^{\prime \prime}, \square\right)$ is a commutative, unital $C^{*}$-algebra, and so has the form $C(\widetilde{K})$ for a compact space $\widetilde{K}$, called the hyper-Stonean envelope of $K$. (See [7] for an extensive discussion and explicit constructions of the space $\widetilde{K}$.) Now suppose that $A$ is a uniform algebra on a non-empty, locally compact space $K$. Then $\left(A^{\prime \prime}, \square\right)$ is also Arens regular and is a closed subalgebra of $C(\widetilde{K})$.

Our results will be based on the following theorem. The proof that (c) implies (d) is surely well-known.

THEOREM 4.1. Let $A$ be a uniform algebra on a non-empty, compact space $K$, and take $x \in K$. Then the following conditions on $x$ are equivalent:

(a) $\varepsilon_{x} \in \operatorname{ex} K_{A}$;

(b) $x \in \Gamma_{0}(A)$;

(c) $M_{x}$ has a $\mathrm{BAI}$;

(d) $M_{x}$ has a CAI.

Proof. The equivalence of (a), (b), and (c) is a special case of [6, Theorem 4.3.5], and trivially (d) implies (c).

Now suppose that (c) holds. Then $M_{x}^{\prime \prime}$ is a closed subalgebra of $C(\widetilde{K})$ and, by [6, Proposition 2.9.16(iii)], $M_{x}^{\prime \prime}$ contains an identity, say e. Clearly $e$ is an idempotent in $C(\widetilde{K})$, and so $|e|_{\widetilde{K}}=1$. By [ [6, Proposition 2.9.16(iii)] again, $M_{x}$ has a CAI, giving (d).

Thus, by Theorem 2.2(i), $M_{x}$ factors whenever $x$ is a $p$-point for a uniform algebra.

Suppose that $A$ is a uniform algebra on a non-empty, compact space $K$ and that $x \in K$ is a peak point, say $f \in A$ peaks at $x$. Then $\left(1_{K}-f^{n}: n \in \mathbb{N}\right)$ is a sequential BAI for $M_{x}$ (with bound 2); set $f_{n}=1_{K}-f^{n}(n \in \mathbb{N}$ ). For each $n \in \mathbb{N}$, there exists $e_{n} \in\left(M_{x}\right)_{[1]}$ with $\left|f_{n}-e_{n} f_{n}\right|_{K}<1 / n$. Now take 
$g \in M_{x}$ and $\varepsilon>0$. Then there exists $n \in \mathbb{N}$ with $\left|g-f_{n} g\right|_{K}<\varepsilon$ and $n \varepsilon>|g|_{K}$, and hence $\left|g-e_{n} g\right|_{K}<2 \varepsilon$. This shows that $\left(e_{n}\right)$ is a sequential CAI for $M_{x}$.

We shall show in Example 4.8(vii) that there is a uniform algebra with a BPAI, but no CPAI, so there is no 'pointwise' analogue of the implication $(\mathrm{c}) \Rightarrow(\mathrm{d})$ of the above theorem.

We now introduce the following definition.

Definition 4.2. Let $A$ be a natural uniform algebra on a non-empty, locally compact space $K$. Then $A$ is a Cole algebra if $\Gamma_{0}(A)=K$.

Of course $C_{0}(K)$ is a Cole algebra.

The reason for this terminology is the following. In the case where $K$ is compact and metrizable, a natural uniform algebra on $K$ is a Cole algebra if and only if every point of $K$ is a peak point. It was a long-standing conjecture, called the 'peak-point conjecture', that $C(K)$ is the only Cole algebra on a compact, metrizable space $K$. The first counter-example is due to Cole [4], and is described in [36, §19]. An example of Basener [1, also described in [36, §19], gives a compact space $K$ in $\mathbb{C}^{2}$ such that the uniform algebra $R(K)$ of all uniform limits on $K$ of the restrictions to $K$ of the functions which are rational on a neighbourhood of $K$, is a Cole algebra, but $R(K) \neq C(K)$. Further, Feinstein [16, 18] obtained examples of non-trivial Cole algebras on compact, metrizable spaces $K$ such that they are a strong Ditkin algebra and are not regular, respectively.

Let $A$ be a natural uniform algebra on the closed unit interval $\mathbb{I}$. Then it is a very famous question of Gel'fand whether $A$ is necessarily equal to $C(\mathbb{I})$. It is a consequence of Rossi's local maximum modulus theorem [36, Corollary 9.14] that $\Gamma_{0}(A)$ is dense in $\mathbb{I}$. However, it seems to be unknown whether every such algebra is necessarily a Cole algebra, and also unknown whether every Cole algebra on $\mathbb{I}$ is necessarily trivial. It is known that strongly regular uniform algebras on $\mathbb{I}$ are trivial [39].

The following theorem is the main result of this section; it is immediate from Theorem 4.1.

TheOREM 4.3. Let $A$ be a uniform algebra on a compact space $K$. Then the following are equivalent:

(a) $A$ is contractive;

(b) A is a Cole algebra;

(c) $M_{x}$ has a BAI for each $x \in K$.

4.2. Pointwise approximate identities for uniform algebras. We now consider when maximal ideals in a uniform algebras have a BPAI or a CPAI and the relationship between 'pointwise contractive' and 'contractive' for uniform algebras. 
Proposition 4.4. Let $A$ be a uniform algebra on a non-empty, locally compact space. Suppose that $A$ has a sequential BPAI. Then $A$ has a sequential CAI.

Proof. There is a sequence $\left(f_{n}\right)$ in $A$ such that $f_{n}(x) \rightarrow 1$ as $n \rightarrow \infty$ for each $x \in K$ and with $\sup \left|f_{n}\right|_{K} \leq m$, say. Take $f \in A$. Then $f f_{n}-f \rightarrow 0$ pointwise on $K$ as $n \rightarrow \infty$, and so, by the dominated convergence theorem,

$$
\lim _{n \rightarrow \infty} \int_{K}\left(f f_{n}-f\right)(x) d \mu(x)=0
$$

for each positive measure $\mu$ on $K$. Thus $\lim _{n \rightarrow \infty}\left\langle f f_{n}-f, \lambda\right\rangle=0$ for each $\lambda \in A^{\prime}$. By [6, Proposition 2.9.14(iii)], $A$ has a BAI of bound $m$. Now $A$ is a maximal ideal in the uniform algebra $A^{\sharp}$ on $K_{\infty}$, and $A$ has a BAI with respect to this uniform norm, and so, by Theorem 4.1, $A$ has a CAI, which we may take to be sequential.

We next recall the definition of a 'Gleason part' for a uniform algebra. The first lemma is [36, Lemma 16.1]; here $\rho$ denotes the hyperbolic metric on $\overline{\mathbb{D}}$.

Lemma 4.5. Let $A$ be a natural uniform algebra on a compact space $K$, and take $x, y \in K$. Then the following are equivalent:

(a) $\left\|\varepsilon_{x}-\varepsilon_{y}\right\|<2$;

(b) there exists $c \in(0,1)$ such that $|f(x)|<c|f|_{K}\left(f \in M_{y}\right)$;

(c) there is a constant $M>0$ such that $\rho(f(x), f(y)) \leq M$ for each $f \in A_{[1]}$.

Now define $x \sim y$ for $x, y \in K$ if $x$ and $y$ satisfy the conditions of the lemma. It follows that $\sim$ is an equivalence relation on $K$; the equivalence classes with respect to this relation are the Gleason parts for $A$. We equip $K$ with the topology induced by the Gleason metric $\delta$, where

$$
\delta(x, y)=\left\|\varepsilon_{x}-\varepsilon_{y}\right\| \quad(x, y \in K) .
$$

The Gleason parts form a partition of $K$, and each part is $\sigma$-compact with respect to the Gleason metric. Clearly $\{x\}$ is a one-point Gleason part whenever $x$ is a $p$-point.

For a discussion of Gleason parts, see [21, Chapter VI].

The following result seems to have been unnoticed so far.

THEOREM 4.6. Let $A$ be a natural uniform algebra on a compact space $K$, and take $x \in K$. Then the following are equivalent:

(a) $\{x\}$ is a one-point Gleason part;

(b) $M_{x}$ has a CPAI;

(c) for each $y \in K \backslash\{x\}$, there is a sequence $\left(f_{n}\right)$ in $M_{x}$ such that $\left|f_{n}\right|_{K} \leq 1(n \in \mathbb{N})$ and $f_{n}(y) \rightarrow 1$ as $n \rightarrow \infty$. 
Proof. The equivalence of (a) and (c) is immediate from Lemma 4.5, and clearly (b) implies (c).

Now suppose that (c) holds, and take a finite set $F=\left\{y_{1}, \ldots, y_{m}\right\}$ in $K \backslash\{x\}$. Take $B$ to be the closed subalgebra of $A$ consisting of the functions in $A$ that are constant on $F$, and set $M=M_{y_{1}} \cap \cdots \cap M_{y_{m}}$, so that $M$ is a maximal ideal of $B$ and $B$ is a natural uniform algebra on the compact set $L$ formed by identifying the points $y_{1}, \ldots, y_{m}$, say this point is $y_{F} \in L$.

For each $j \in \mathbb{N}_{m}$, there is a sequence $\left(f_{j, n}: n \in \mathbb{N}\right)$ in $\left(M_{y_{j}}\right)_{[1]}$ with $\lim _{n \rightarrow \infty} f_{j, n}(x)=1$. Set

$$
f_{n}=f_{1, n} \cdots f_{m, n} \quad(n \in \mathbb{N}) .
$$

Then $f \in M$ and $\lim _{n \rightarrow \infty} f_{n}(x)=1$, and so $x \nsim y_{F}$ in $L$. Hence there exists $\left(h_{n}\right)$ in $\left(M_{x}\right)_{[1]}$ with $\lim _{n \rightarrow \infty} h_{n}\left(y_{F}\right)=1$. We see that

$$
\lim _{n \rightarrow \infty} h_{n}\left(y_{j}\right)=1 \quad\left(j \in \mathbb{N}_{m}\right)
$$

as sequence in $A$, and so $M_{x}$ has a CPAI, giving (b).

Let $A$ be a natural uniform algebra on a compact space $K$, and take $x \in K$. Suppose that $M_{x}$ has a BPAI. Then $x$ is isolated in $K$ with respect to the Gleason metric.

The following immediate consequence of the above theorem is the second main result of this section.

THEOREM 4.7. Let $A$ be a natural uniform algebra on a compact space $K$. Then $A$ is pointwise contractive if and only if each Gleason part in $K$ is a singleton.

We now present various uniform algebras; they show that all possibilities not excluded by previous theorems do occur.

ExAmple 4.8. (i) Let $A=A(\overline{\mathbb{D}})$ be the disc algebra, as in Example 2.4 . and, for $z \in \overline{\mathbb{D}}$, set $M_{z}=\{f \in A: f(z)=0\}$. Take $z \in \mathbb{D}$ and $f \in M_{z}$. By the Schwarz-Pick theorem,

$$
|f(w)| \leq \frac{|w-z|}{|1-\bar{w} z|}|f|_{\overline{\mathbb{D}}} \quad(w \in \mathbb{D}) .
$$

Thus there exists $\delta>0$ such that $|f(w)|<1 / 2$ whenever $|w-z|<\delta$, and so $M_{z}$ does not have a BPAI.

Take $z \in \overline{\mathbb{D}}$. In this example, $M_{z}$ has a BPAI if and only if $M_{z}$ has a CPAI if and only if $z$ is a peak point.

(ii) Let $A$ be a uniform algebra on a compact set $K$, and take $x \in K$. It is also possible to have $x \in \Gamma(A)$, but such that $M_{x}$ does not have a BPAI. Indeed, let $K=\overline{\mathbb{D}} \times \mathbb{I}$ and take $A$ to be the 'tomato can algebra' [36, Example 7.8], so that $A$ is the uniform algebra of all continuous functions $f$ 
on $K$ such that the function $z \mapsto f(z, 1), \overline{\mathbb{D}} \rightarrow \mathbb{C}$, belongs to $A(\overline{\mathbb{D}})$. Then

$$
\Gamma_{0}(A)=\{(z, t) \in K: 0 \leq t<1\} \cup\{(z, 1) \in K: z \in \mathbb{T}\}
$$

and $\Gamma(A)=K$. The set $K \backslash \Gamma_{0}(A)=\{(z, 1): z \in \mathbb{D}\}$ is a Gleason part, and again we see that $M_{x}$ has a BPAI if and only if $M_{x}$ has a CPAI if and only if $x$ is a peak point, where $x \in K$; if $x \in K \backslash \Gamma_{0}(A)$, then $M_{x}^{2}=\overline{M_{x}^{2}} \subsetneq M_{x}$, and so $M_{x}$ does not have an approximate identity.

(iii) Let $K$ be a compact plane set, and consider the natural uniform algebra $R(K)$ on $K$. Take $x \in K$. Then $\{x\}$ is a one-point Gleason part if and only $x$ is a peak point [36, Corollary 26.14], and so $M_{x}$ has a BAI if and only if $M_{x}$ has a CPAI if and only if $x$ is a peak point for $R(K)$. By [36. Corollary 26.15], $R(K)=C(K)$ if and only if each point of $K$ is a onepoint Gleason part, and so $R(K)=C(K)$ if and only if $R(K)$ is pointwise contractive. It follows from [36, Corollary 26.12] that $x$ is not isolated in the Gleason metric whenever $x$ is not a peak point, and so $M_{x}$ has a BPAI if and only if it has a CPAI. (By [36, Corollary 26.13] each Gleason part for $R(K)$ that is not a singleton has positive plane area.)

(iv) There are natural, separable uniform algebras $A$ on a compact space $K$ that have one-point parts $\{x\}$ for some $x \in K \backslash \Gamma(A)$. For such points $x$, the maximal ideal $M_{x}$ has a CPAI, but no BAI. For example, the uniform algebra $\mathfrak{A}_{\alpha}$ of [36, Theorem 18.1] has this property.

(v) Let $H^{\infty}$ be the uniform algebra of all bounded analytic functions on $\mathbb{D}$, so that $H^{\infty}$ is non-separable. The (large) character space of $H^{\infty}$ is denoted by $\Phi$; it is studied in [22, Chapter 10]. Since $H^{\infty}$ is a logmodular algebra on its Šilov boundary, every point of $\Gamma=\Gamma\left(H^{\infty}\right)$ has a unique representing measure on $\Gamma$, and this unique representing measure must be the point mass. Consequently, each point of $\Gamma$ is a $p$-point, and hence a one-point Gleason part. In fact, each Gleason part for $H^{\infty}$ is either a onepoint part or an analytic disc and there are one-point Gleason parts that are not in $\Gamma\left(H^{\infty}\right)$. Suppose that $\{x\}$ is a one-point part with $x \in \Phi$. Then $M_{x}$ factors [22, Theorem 2.4].

(vi) The following example is given in [8, Theorem 2.3]. There is a natural, separable uniform algebra on a compact, metric space $K$ such that each point of $K$ is a one-point Gleason part, so that $A$ is pointwise contractive, but $\Gamma(A) \subsetneq K$, so that $A$ is not a Cole algebra, and hence not contractive. Thus some maximal ideals of $A$ are pointwise contractive uniform algebras without a BAI. The existence of such an example also follows from Theorem 2.5 in [4].

(vii) In [17, Theorem 2.1], Feinstein constructed a separable, regular, natural uniform algebra $A$ on a compact space $K$ such that there is a twopoint Gleason part, say $\left\{x_{1}, x_{2}\right\}$, and such that all other points of $K$ are one-point Gleason parts. 
Set $M=M_{x_{1}}$, and take a finite set $F$ in $K \backslash\left\{x_{1}\right\}$, say $F$ is a subset of a set of the form $\left\{x_{2}, \ldots, x_{n}\right\}$, where $x_{2}, \ldots, x_{n}$ are distinct. Take $f_{2} \in M_{x_{2}}$ with $f_{2}\left(x_{1}\right)=1$, and set $m=\left|f_{2}\right|_{K}$. Fix $\varepsilon \in(0,1)$, and take $\delta>0$ such that $m n \delta<\varepsilon$. For $j=3, \ldots, n$, take $f_{j} \in M_{x_{j}}$ with $\left|f_{j}\left(x_{1}\right)\right|>1-\delta$ and $\left|f_{j}\right|_{K}=1$, and set $f=f_{2} f_{3} \cdots f_{n} \in A$, so that $|f|_{K} \leq m$ and $\left|f\left(x_{1}\right)-1\right|<m n \delta<\varepsilon$. Finally, set $g=f\left(x_{1}\right) 1_{K}-f$, so that $g \in M$ with $\left|g\left(x_{j}\right)\right|>1-\varepsilon$ and $|g|_{K} \leq m+1+\varepsilon$. It follows that $M$ has a BPAI with bound $m+1$. Similarly, $M_{x_{2}}$ has a BPAI, and each other point of $K$ has a CPAI.

Thus, in this example, each maximal ideal has a BPAI (with a uniform bound), but the algebra is not pointwise contractive.

(viii) In Examples 5.13 and 5.16 of [35], there are natural uniform algebras $A$ on compact spaces $K$ and points $x \in K \backslash \Gamma(A)$ such that $\{x\}$ is a one-point Gleason part and $M_{x}^{2}$ is not dense in $M_{x}$; in particular, $M_{x}$ does not factor. In these cases, the uniform algebra $M_{x}$ has a CPAI, but no approximate identity. We are grateful to Alexander Izzo for pointing out this example.

We are grateful to Joel Feinstein for pointing out the following example. We obtain a strong form of a CPAI in a maximal ideal $M_{x}$ such that, for each non-empty, finite set $F$ disjoint from $x$, there is a function $f \in\left(M_{x}\right)_{[1]}$ that attains the value 1 at each point in $F$.

THEOREM 4.9. There is a natural, pointwise contractive uniform algebra $A$ on a non-empty, compact, metrizable space $K$ such that a maximal ideal $M$ of $A$ does not have a BAI, and $\Gamma_{0}(A) \subsetneq K$.

Proof. Let $A$ be the uniform algebra on a compact, metrizable space $K$ that is constructed in [16, Theorem 5.1]: the algebra $A$ is natural and has the property that there exists a point $x \in K$ such that $\Gamma_{0}(A)=K \backslash\{x\}$, and so $A \neq C(K)$.

Let $F$ be a non-empty, finite subset of $K \backslash\{x\}$. Then $F$ is a peak set, and so there exists a function $f \in A$ such that $f(y)=1(y \in F)$ and $|f(y)|<1$ $(y \in K \backslash F)$. Set $a=f(x)$, so that $a \in \mathbb{D}$. By composing $f$ with the function $\psi_{a}(z)=\zeta(z-a) /(1-\bar{a} z)(z \in \overline{\mathbb{D}})$ for suitable $\zeta \in \mathbb{T}$, we may suppose that $f \in M_{x}$. Thus $M_{x}$ has a CAI. Each point $y \in K \backslash\{x\}$ is a peak point for $A$, and so $M_{y}$ has a CAI. Thus $A$ is pointwise contractive.

Since $x \notin \Gamma_{0}(A)$, it follows from Theorem 4.1 that $M_{x}$ does not have a BAI. Thus $A$ is not contractive, and $A$ is not a Cole algebra.

We do not know whether there is a natural uniform algebra $A$ on a compact space $K$ such that every point of $K$ is a one-point part, but $M$ does not have an approximate identity for some maximal ideal $M$ in $A$. 
5. Banach function algebras on closed intervals. We now present two natural, unital Banach function algebras on intervals of $\mathbb{R}$. The first example gives a pointwise contractive Ditkin algebra such that one maximal ideal does not have BRAUs, and hence has no BAI. This maximal ideal is an abstract Segal algebra with respect to $C_{0}((0,1])$. The example is easier than that of Jones and Lahr, and a small variation is also stronger in that it is pointwise contractive, but has no approximate identity. The example is developed from a suggestion of Charles Read.

The second example exhibits a contractive Banach function algebra that is not equivalent to a uniform algebra.

Example 5.1. Consider the set $A$ of functions $f \in C(\mathbb{I})$ such that

$$
I(f):=\int_{0}^{1} \frac{|f(t)-f(0)|}{t} d t<\infty .
$$

Clearly $A$ is a self-adjoint, linear subspace of $C(\mathbb{I})$ containing the polynomials, and so $A$ is uniformly dense in $C(\mathbb{I})$. Indeed, $A$ is 'large', in that it contains all the Banach function algebras $\left(\operatorname{Lip}_{\alpha}(\mathbb{I}),\|\cdot\|_{\alpha}\right)($ for $0<\alpha \leq 1)$. Also, $A$ contains each $f \in C(\mathbb{I})$ with supp $f \subset(0,1]$.

For $f \in A$, define

$$
\|f\|=|f|_{\mathbb{I}}+I(f) .
$$

Clearly $(A,\|\cdot\|)$ is a normed space; we recall a standard fact that it is complete. Indeed, take $\left(f_{n}\right)$ to be a Cauchy sequence in $(A,\|\cdot\|)$. Then there exists $f \in C(\mathbb{I})$ such that $\left|f_{n}-f\right|_{\mathbb{I}} \rightarrow 0$ as $n \rightarrow \infty$. Take $\varepsilon>0$. Then there exists $n_{0} \in \mathbb{N}$ such that

$$
\left|f_{m}-f_{n}\right|_{I}+I\left(f_{m}-f_{n}\right)<\varepsilon \quad\left(m, n \geq n_{0}\right) .
$$

By Fatou's lemma, $I\left(f_{m}-f\right) \leq \liminf _{n \rightarrow \infty} I\left(f_{m}-f_{n}\right) \leq \varepsilon$ for each $m \geq n_{0}$. We see that $I(f) \leq I\left(f_{n_{0}}\right)+\varepsilon$, and so $f \in A$; further,

$$
\left\|f_{m}-f\right\| \leq 2 \varepsilon \quad\left(m \geq n_{0}\right),
$$

and hence $\left(f_{n}\right)$ converges to $f$ in $(A,\|\cdot\|)$.

Our first claim is that the set $A$ is a subalgebra of $C(\mathbb{I})$ and, further, that $\|f g\| \leq\|f\|\|g\|(f, g \in A)$. Indeed, for $f, g \in A$, we have

$$
|(f g)(t)-(f g)(0)| \leq|f|_{\mathbb{I}}|g(t)-g(0)|+|g|_{\mathbb{I}}|f(t)-f(0)| \quad(t \in \mathbb{I}),
$$

and so $I(f g) \leq|f|_{\mathbb{I}} I(g)+|g|_{\mathbb{I}} I(f)$, which implies the claim. Also, $\left\|1_{\mathbb{I}}\right\|=1$, and so $(A,\|\cdot\|)$ is a Banach function algebra on $\mathbb{I}$. Set $M=M_{0}(A)$. Then $M$ is also an ideal in $C_{0}((0,1])$ and

$$
\|f g\| \leq\|f\||g|_{\mathbb{I}} \quad\left(f \in M, g \in C_{0}((0,1])\right) .
$$

Our second claim is that $A$ is natural on II. By [6, Proposition 4.1.5(ii)], it suffices to show that each $f \in A$ that is such that $f(t) \neq 0(t \in \mathbb{I})$ is 
invertible in $A$. We may suppose, without loss of generality, that $f(0)=1$, say $f=1+g$, where $g \in M$, and that $1 / f=1+h$, where $h \in C(\mathbb{I})$ and $h(0)=0$. Choose $\delta>0$ such that $|g(t)|<1 / 2(t \in[0, \delta])$. Since $|h(t)| \leq 2|g(t)|(t \in[0, \delta])$, we have $\int_{0}^{\delta}(|h(t)| / t) d t<\infty$; clearly,

$$
\int_{\delta}^{1} \frac{|h(t)|}{t} d t \leq|h|_{\mathbb{I}} \log (1 / \delta)<\infty,
$$

and so $h \in M$ and $1 / f \in A$, giving the claim.

Our third claim is that the norm of $A$ is not equivalent to the uniform norm. To see this, take $n \in \mathbb{N}$, and define $f_{n}$ to be linear on $[0,1 / n]$ and equal to 1 on $[1 / n, 1]$. Then $f_{n} \in A$ and

$$
\left\|f_{n}\right\|=1+\int_{0}^{1 / n} n d t+\int_{1 / n}^{1} \frac{1}{t} d t=2+\log n,
$$

whereas $\left|f_{n}\right|_{\mathbb{I}}=1$. This gives the claim. Alternatively, take

$$
h_{0}(t)=\frac{1}{\log (1 / t)} \quad(t \in(0,1]),
$$

with $h_{0}(0)=0$. Then $h_{0} \in C(\mathbb{I})$, but $h_{0} \notin A$. Indeed, for each $n \in \mathbb{N}$ and $f \in M$ with

$$
|1-f(x)|<1 / 2 \quad(1 / n \leq x \leq 1),
$$

we have $\|f\| \geq(\log n) / 2$, and so $M$ does not have BRAUs.

Take $t_{0} \in \mathbb{I}$, and take $\left(g_{n}\right)$ to be as in Definition 2.5, so that $\left(g_{n}\right)$ is a sequence in $J_{t_{0}}(A)$, and take $f \in M_{t_{0}}$. We claim that $\left\|f-f g_{n}\right\| \rightarrow 0$ as $n \rightarrow \infty$. This is immediate for $t_{0}>0$. In the case where $t_{0}=0$, fix $\varepsilon>0$ and take $\delta>0$ such that $|f(t)|<\varepsilon(0 \leq t \leq \delta)$ and

$$
\int_{0}^{\delta} \frac{|f(t)|}{t} d t<\varepsilon .
$$

Then, for $n>1 / \delta$, we see that $\left\|f-f g_{n}\right\|<2 \varepsilon$. The claim follows. Thus the natural Banach function algebra $A$ is a Ditkin algebra, and hence strongly regular. The sequence $\left(g_{n}\right)$ (for $t_{0}=0$ ) is an approximate identity for $M$ and $C_{0}((0,1])$, and so $M$ is an abstract Segal algebra with respect to $C_{0}((0,1])$. For $t_{0}>0$, we see that $\left\|g_{n}\right\|=1+O(1 / n)$ as $n \rightarrow \infty$, and so the sequence $\left(g_{n} /\left\|g_{n}\right\|: n \in \mathbb{N}\right)$ is a CAI for $M_{t_{0}}$. This implies that $M_{t_{0}}=M_{t_{0}}^{[2]}=\bar{J}_{t_{0}}$.

Our fourth claim is that the algebra of polynomials (restricted to $\mathbb{I}$ ) is dense in $A$. Indeed, to see this, it suffices to show that, given $f \in J_{0}$ and $\varepsilon>0$, there is a polynomial $p$ such that $\|f-p\|<\varepsilon$. For this, we first define $g(t)=f(t) / t(t \in \mathbb{I})$ (with $g(0)=0)$, so that $g \in C(\mathbb{I})$. There is a 
polynomial $q$ such that $|g-q|_{\mathbb{I}}<\varepsilon / 2$. Set $p(t)=t q(t)(t \in \mathbb{I})$, so that $p$ is a polynomial; clearly $\|f-p\|<\varepsilon$, as required.

The ideal $M$ does not have a BAI. Indeed, our fifth claim is the slightly stronger fact that $M^{2}$ has infinite codimension in $M$, and so the space of point derivations at 0 is infinite dimensional. To see this, first take $f \in M^{2}$, say $f=\sum_{j=1}^{k} g_{j} h_{j}$, where $g_{1}, \ldots, g_{k}, h_{1}, \ldots, h_{k} \in M$, and set

$$
u=\sum_{j=1}^{k}\left(\left|g_{j}\right|+\left|h_{j}\right|\right) .
$$

Then $u \in M$ and $|f(t)| \leq u(t)^{2}(t \in \mathbb{I})$. We apply this with $f_{\alpha}$ defined by

$$
f_{\alpha}(t)=\frac{1}{(\log (1 / t))^{\alpha}} \quad(t \in(0,1]),
$$

with $f_{\alpha}(0)=0$, for $\alpha>0$. Then $f_{\alpha} \in M$ whenever $\alpha>1$. Suppose that $f_{\alpha} \in M^{2}$. Then there exists $u_{\alpha} \in M$ such that $\left|f_{\alpha}(t)\right| \leq u_{\alpha}(t)^{2}(t \in \mathbb{I})$, and so $u_{\alpha}(t) \geq 1 /(\log (1 / t))^{\alpha / 2}(t \in(0,1])$. This implies that $\alpha>2$, and so $f_{\alpha} \in M \backslash M^{2}$ for $\alpha \in(1,2]$. It follows easily that the set

$$
\left\{f_{\alpha}+M^{2}: \alpha \in(1,2]\right\}
$$

is linearly independent in $M / M^{2}$, giving the claim.

We have seen that the maximal ideal $M$ does not have a BAI. However, our sixth claim is that it has a CPAI. Indeed, take $F$ to be a finite subset of $(0,1]$, say $F=\left\{t_{1}, \ldots, t_{k}\right\}$. For each $n \in \mathbb{N}$ and $i \in \mathbb{N}_{k}$, take $f_{n, i}$ to be the restriction to $\mathbb{I}$ of the function such that $f_{n, i}\left(x_{i}\right)=1$, such that $f_{n, i}=0$ outside the interval $\left[t_{i}-1 / n, t_{i}+1 / n\right]$, and such that $f_{n, i}$ is linear on $\left[t_{i}-1 / n, t_{i}\right]$ and $\left[t_{i}, t_{i}+1 / n\right]$, and set $f_{F, n}=\sum_{i=1}^{k} f_{n, i}$. Then clearly $f_{F, n}(t)=1(t \in F)$ and $\left\|f_{F, n}\right\|=1+O(1 / n)$ as $n \rightarrow \infty$. The claim follows. (We note that our CPAI in $M$ is a net, not a sequence.) It follows that $A$ is pointwise contractive.

Define $h_{0}$ as in equation (5.1) above, so that $h_{0} \in C(\mathbb{I}) \backslash M$ and $h_{0}^{2} \in M$, and then set $B=M \oplus \mathbb{C} h_{0}$. We have $\left\|f h_{0}\right\| \leq\|f\|\left|h_{0}\right|_{\mathbb{I}}(f \in M)$, and so, by multiplying $h_{0}$ by a suitable positive constant, we may suppose that $h_{0}$ satisfies the conditions of Proposition 3.8. Since $M$ is pointwise contractive, so is $B$. However, $B$ does not have any approximate identity.

EXAMPLE 5.2. We now present a Banach function algebra on the circle $\mathbb{T}$, but, for notational convenience, we identify $C(\mathbb{T})$ with the subalgebra of $C([-1,1])$ consisting of functions $f \in C([-1,1])$ with

$$
\exp (-\pi i f(-1))=\exp (\pi i f(1)) .
$$

Addition and subtraction in $[-1,1]$ are taken modulo $[-1,1]$.

We fix a constant $\alpha$ with $1<\alpha<2$. 
For $t \in[-1,1]$, the shift of $f \in C(\mathbb{T})$ by $t$ is defined by

$$
\left(S_{t} f\right)(s)=f(s-t) \quad(s \in[-1,1]) ;
$$

the oscillation of $f$ is

$$
\omega_{f}(t)=\sup \left\{\left|f(s)-\left(S_{t} f\right)(s)\right|: s \in[-1,1]\right\} \quad(t \in[-1,1]),
$$

and

$$
\Omega_{f}(t)=\left\|f-S_{t} f\right\|_{1}=\int_{-1}^{1}|f(s)-f(s-t)| d s \quad(t \in[-1,1]) .
$$

Then we define

$$
I(f)=\int_{-1}^{1} \frac{\Omega_{f}(t)}{|t|^{\alpha}} d t
$$

We note that the function $t \mapsto \Omega_{f}(t),[-1,1] \rightarrow \mathbb{R}^{+}$, is continuous, and so $I(f)$ is well defined (in $[0, \infty]$ ). Also $\Omega_{f}=\Omega_{1-f}$, and so $I(f)=I(1-f)$.

We now define $A$ to be the space of functions $f \in C(\mathbb{T})$ with $I(f)<\infty$, and set

$$
\|f\|=|f|_{\mathbb{T}}+I(f) \quad(f \in A) .
$$

Clearly $A$ is a self-adjoint, linear subspace of $C(\mathbb{T})$, and $(A,\|\cdot\|)$ is a normed space. Further, $(A,\|\cdot\|)$ is complete, and so is a Banach space. For let $\left(f_{n}\right)$ be a Cauchy sequence in $(A,\|\cdot\|)$. Then there exists $f \in C(\mathbb{T})$ with $f_{n} \rightarrow f$ uniformly on $\mathbb{T}$ as $n \rightarrow \infty$. We have $\Omega_{f_{n}}(t) \rightarrow \Omega_{f}(t)$ as $n \rightarrow \infty$ for each $t \in[-1,1]$, and so it again follows from Fatou's lemma that $f_{n} \rightarrow f$ in $(A,\|\cdot\|)$ as $n \rightarrow \infty$.

We see that $S_{t} f \in A$ with $\left\|S_{t} f\right\|=\|f\|$ for each $f \in A$ and $t \in[-1,1]$, and so the algebra $A$ is homogeneous on the circle.

Suppose that $f \in C(\mathbb{T})$ with $\omega_{f}(t)=O\left(t^{\gamma}\right)$ for some $\gamma>\alpha-1$. Then $f \in A$. In particular, $A$ contains the trigonometric polynomials, and so $A$ is uniformly dense in $C(\mathbb{T})$.

Our first claim is that $(A,\|\cdot\|)$ is a Banach function algebra on $\mathbb{T}$. Indeed, take $f, g \in A$ and $t \in[-1,1]$. For each $s \in[-1,1]$, we have

$$
\left|\left(f g-S_{t}(f g)\right)(s)\right| \leq|f|_{\mathbb{T}}\left|\left(g-S_{t} g\right)(s)\right|+|g|_{\mathbb{T}}\left|\left(f-S_{t} f\right)(s)\right|,
$$

and so $\Omega_{f g}(t) \leq|f|_{\mathbb{T}} \Omega_{g}(t)+|g|_{\mathbb{T}} \Omega_{f}(t)$. It follows that

$$
I(f g) \leq|f|_{\mathbb{T}} I(g)+|g|_{\mathbb{T}} I(f) \text { and hence } \quad\|f g\| \leq\|f\|\|g\| .
$$

Further, $\left\|1_{\mathbb{T}}\right\|=1$. The claim follows.

Our second claim is that $A$ is natural on $[-1,1]$. Indeed, take a function $f \in A$ such that $|f(t)| \geq \delta>0(t \in[-1,1])$. Then we see easily that $I(1 / f) \leq \delta^{2} I(f)<\infty$, and so $1 / f \in A$. Thus $A$ is natural.

Our third claim is that $A \neq C(\mathbb{T})$. For this, we define

$$
e_{n}(s)=\exp (i \pi n s) \quad(s \in[-1,1])
$$


for $n \in \mathbb{N}$. Then, for each $n \in \mathbb{N}$, we have

$$
\Omega_{e_{n}}(t)=\int_{-1}^{1}\left|e^{i \pi n s}-e^{i \pi n(s-t)}\right| d s=2\left|1-e^{i \pi n t}\right| \quad(t \in[-1,1]),
$$

and so

$$
I\left(e_{n}\right)=2 \int_{-1}^{1} \frac{\left|1-e^{i \pi n t}\right|}{|t|^{\alpha}} d t \geq 2(\pi n)^{\alpha-1} \int_{\pi / 2}^{\pi} \frac{\left|1-e^{i u}\right|}{|u|^{\alpha}} d u=C n^{\alpha-1}
$$

for some constant $C>0$. Thus $\left\|e_{n}\right\| \geq C n^{\alpha-1} \rightarrow \infty$ as $n \rightarrow \infty$, whereas $\left|e_{n}\right|_{\mathbb{T}}=1$ for each $n \in \mathbb{N}$, and so the claim follows.

Our fourth claim is that the Banach function algebra $A$ is contractive. Since $A$ is homogeneous on $\mathbb{T}$, it suffices to show that the maximal ideal $M:=\{f \in A: f(0)=0\}$ has a CAI. For this, define

$$
\Delta_{n}(s)=\max \{1-n|s|, 0\} \quad(s \in[-1,1], n \in \mathbb{N}) .
$$

Take $n \in \mathbb{N}$. Suppose first that $|t| \leq 1 / n$. Then $\left|\Delta_{n}(s)-\Delta_{n}(s-t)\right| \leq n|t|$ for $|s| \leq 2 / n$ and $\Delta_{n}(s)=\Delta_{n}(s-t)=0$ for $|s| \geq 2 / n$, and so

$$
\Omega_{\Delta_{n}}(t) \leq 2 \int_{0}^{2 / n} n|t| d s=4|t|
$$

Second, suppose that $|t| \geq 1 / n$. Then

$$
\Omega_{\Delta_{n}}(t) \leq 2 \int_{-1}^{1} \Delta_{n}(s) d s=\frac{2}{n}
$$

Hence

$$
I\left(\Delta_{n}\right) \leq 8 \int_{0}^{1 / n} t^{1-\alpha} d t+\frac{2}{n} \int_{1 / n}^{1} t^{-\alpha} d t=O\left(\frac{1}{n^{2-\alpha}}\right) \rightarrow 0 \quad \text { as } n \rightarrow \infty
$$

because $\alpha<2$, and so $\left\|1-\Delta_{n}\right\|=\left\|\Delta_{n}\right\|=1+o(1)$ as $n \rightarrow \infty$.

In fact, we can suppose that $I\left(\Delta_{n}\right) \leq 1$ and $\left\|\Delta_{n}\right\| \leq 2$ for all $n \in \mathbb{N}$.

Finally, we show that $\left(1-\Delta_{n}: n \in \mathbb{N}\right)$ is an approximate identity for the maximal ideal $M$. Certainly $1-\Delta_{n} \in M(n \in \mathbb{N})$. Now write

$$
I_{\delta}(f)=\int_{-\delta}^{\delta} \frac{\Omega_{f}(t)}{|t|^{\alpha}} d t, \quad J_{\delta}(f)=2 \int_{\delta}^{1} \frac{\Omega_{f}(t)}{|t|^{\alpha}} d t \quad(f \in A)
$$

for each $\delta>0$, so that $I(f)=I_{\delta}(f)+J_{\delta}(f)(f \in A)$. Fix $f \in M$ and $\varepsilon>0$, and then choose $\delta>0$ such that $I_{\delta}(f)<\varepsilon$ and $|f|_{[-\delta, \delta]}<\varepsilon$. Then

$$
I_{\delta}\left(f \Delta_{n}\right) \leq\left|\Delta_{n}\right|_{\mathbb{T}} I_{\delta}(f)+|f|_{[-\delta, \delta]} I\left(\Delta_{n}\right)<2 \varepsilon .
$$


Next choose $n_{0} \in \mathbb{N}$ such that $n_{0} \delta>1$ and

$$
\int_{-1 / n_{0}}^{1 / n_{0}}|f(t)| d t<\varepsilon \delta^{\alpha} .
$$

Take $n \geq n_{0}$. Then $\left|f \Delta_{n}\right|_{[-1,1]} \leq|f|_{[-\delta, \delta]}<\varepsilon$ and

$$
\Omega_{f \Delta_{n}}(t) \leq 2 \int_{-1 / n}^{1 / n}|f(s)| d s<2 \varepsilon \delta^{\alpha} \quad(t \in[-1,1]),
$$

and so

$$
J_{\delta}\left(f \Delta_{n}\right)<4 \varepsilon \delta^{\alpha} \int_{\delta}^{1} \frac{d t}{t^{\alpha}}<4 \varepsilon .
$$

Hence $\left\|f \Delta_{n}\right\| \leq \varepsilon+2 \varepsilon+4 \varepsilon=7 \varepsilon\left(n \geq n_{0}\right)$. Thus $f \cdot\left(1-\Delta_{n}\right) \rightarrow f$ in $(A,\|\cdot\|)$ as $n \rightarrow \infty$, so that $\left(1-\Delta_{n}: n \in \mathbb{N}\right)$ is indeed an approximate identity for $M$.

We conclude that $\left(\left(1-\Delta_{n}\right) /\left\|1-\Delta_{n}\right\|: n \in \mathbb{N}\right)$ is a CAI in $M$, and so $A$ is contractive.

The above example is somewhat related to the 'remarkable homogeneous Banach algebra' of Pisier [32, as discussed in [31. In that example, in the formulation of [31, Theorem 2.1], $\Omega_{f}(t)$ is replaced by $\Psi_{f}(t)$, where $\Psi_{f}$ is the increasing rearrangement of the function $t \mapsto\left\|f-S_{t} f\right\|_{2}$, and the function $1 / t^{\alpha}$ on $(0,1]$ is replaced by $1 / t(\log (1 / t))^{1 / 2}$. However, this example is not contractive.

Acknowledgements. The authors thank David Blecher, Joel Feinstein, Alexander Izzo, and Charles Read for enjoyable and informative conversations. The first author is grateful to the second author and Koç University for generous hospitality on two occasions.

\section{References}

[1] R. F. Basener, On rationally convex hulls, Trans. Amer. Math. Soc. 182 (1973), 353-381.

[2] D. Blecher and C. J. Read, Operator algebras with contractive approximate identities, IV: a large operator algebra in $c_{0}$, Trans. Amer. Math. Soc., to appear.

[3] C. Chou and G. Xu, The weak closure of the set of left translation operators, Proc. Amer. Math. Soc. 127 (1999), 465-471.

[4] B. Cole, One point parts and the peak point conjecture, Thesis, Yale Univ., 1968.

[5] H. G. Dales, Boundaries and peak points for Banach function algebras, Proc. London Math. Soc. (3) 22 (1971), 121-136.

[6] H. G. Dales, Banach Algebras and Automatic Continuity, London Math. Soc. Monogr. 24, Clarendon Press, Oxford, 2000. 
[7] H. G. Dales, F. K. Dashiell, Jr., A. T.-M. Lau, and D. Strauss, Banach Spaces of Continuous Functions as Dual Spaces, CMS Books in Math./Ouvrages de Math. de la SMC, Springer, New York, to appear.

[8] H. G. Dales and J. F. Feinstein, Banach function algebras with dense invertible group, Proc. Amer. Math. Soc. 136 (2008), 1295-1304.

[9] H. G. Dales and A. T.-M. Lau, The second duals of Beurling algebras, Mem. Amer. Math. Soc. 177 (2005), no. 836, 191 pp.

[10] H. G. Dales, A. T.-M. Lau, and D. Strauss, Banach algebras on semi-groups and on their compactifications, Mem. Amer. Math. Soc. 205 (2010), no. 966, 165 pp.

[11] H. G. Dales, A. T.-M. Lau, and D. Strauss, Second duals of measure algebras, Dissertationes Math. 481 (2012), 121 pp.

[12] H. G. Dales and R. J. Loy, Approximate amenability of semi-group algebras and Segal algebras, Dissertationes Math. 474 (2010), 58 pp.

[13] H. G. Dales and A. Ülger, Banach function algebras having a BSE norm, in preparation.

[14] J. Delaporte and A. Derighetti, Best bounds for the approximate units for certain ideals of $L^{1}(G)$ and of $A_{p}(G)$, Proc. Amer. Math. Soc. 124 (1996), 1159-1169.

[15] P. Eymard, L'algèbre de Fourier d'un groupe localement compact, Bull. Soc. Math. France 92 (1964), 181-236.

[16] J. F. Feinstein, A non-trivial, strongly regular uniform algebra, J. London Math. Soc. (2) 45 (1992), 288-300.

[17] J. F. Feinstein, Regularity conditions for Banach function algebras, in: Function Spaces (Edwardsville, IL, 1994), Lecture Notes in Pure Appl. Math. 172, Dekker, New York, 1995, 117-122.

[18] J. F. Feinstein, Trivial Jensen measures without regularity, Studia Math. 148 (2001), $67-74$.

[19] B. E. Forrest, E. Kaniuth, A. T.-M. Lau, and N. Spronk, Ideals with bounded approximate identities in Fourier algebras, J. Funct. Anal. 203 (2003), 286-304.

[20] B. Forrest and N. Spronk, Best bounds for approximate identities in ideals of the Fourier algebra vanishing on subgroups, Proc. Amer. Math. Soc. 134 (2005), 111116.

[21] T. W. Gamelin, Uniform Algebras, Prentice-Hall, Englewood Cliffs, NJ, 1969.

[22] J. B. Garnett, Bounded Analytic Functions, Academic Press, San Diego, CA, 1981.

[23] S. Grabiner, Weighted shifts and Banach algebras of power series, Amer. J. Math. 97 (1975), 16-42.

[24] E. Hewitt and H. S. Zuckerman, Singular measures with absolutely continuous convolution squares, Proc. Cambridge Philos. Soc. 62 (1966), 399-420; Corrigendum, ibid. 63 (1967), 367-368.

[25] J. Inoue and S.-E. Takahasi, Constructions of bounded weak approximate identities for Segal algebras on LCA groups, Acta Sci. Math. (Szeged) 66 (2000), 257-271.

[26] J. Inoue and S.-E. Takahasi, On characterizations of the image of the Gelfand transform of commutative Banach algebras, Math. Nachr. 280 (2007), 105-126.

[27] C. A. Jones and C. D. Lahr, Weak and norm approximate identities are different, Pacific J. Math. 72 (1977), 99-104.

[28] E. Kaniuth and A. T. Lau, A separation property of positive definite functions on locally compact groups and applications to Fourier algebras, J. Funct. Anal. 175 (2000), 89-110.

[29] E. Kaniuth and A. Ülger, The Bochner-Schoenberg-Eberlein property for commutative Banach algebras, especially Fourier and Fourier-Stietltjes algebras, Trans. Amer. Math. Soc. 362 (2010), 4331-4356. 
[30] T. W. Palmer, Banach Algebras and the General Theory of *-Algebras. Volume 1: Algebras and Banach Algebras, Encyclopedia Math. Appl. 49, Cambridge Univ. Press, 1994.

[31] T. V. Pedersen, Some properties of the Pisier algebra, Math. Proc. Cambridge Philos. Soc. 128 (2000), 343-354.

[32] G. Pisier, A remarkable homogeneous Banach algebra, Israel J. Math. 34 (1979), $38-44$.

[33] J. S. Pym, The convolution of functionals on spaces of bounded functions, Proc. London Math. Soc. (3) 15 (1965), 84-104.

[34] W. Rudin, Fourier Analysis on Groups, Wiley, New York, 1962.

[35] S. J. Sidney, Properties of the sequence of closed powers of a maximal ideal in a sup-norm algebra, Trans. Amer. Math. Soc. 131 (1968), 128-148.

[36] E. L. Stout, The Theory of Uniform Algebras, Bogden and Quigley, Tarrytown-onHudson, New York, 1971.

[37] S.-E. Takahasi and O. Hatori, Commutative Banach algebras which satisfy a Bochner-Schoenberg-Eberlein-type theorem, Proc. Amer. Math. Soc. 110 (1990), 149-158.

[38] A. Ülger, Some results about the spectrum of commutative Banach algebras under the weak topology and applications, Monatsh. Math. 121 (1996), 353-379.

[39] D. R. Wilken, A note on strongly regular function algebras, Canad. J. Math. 21 (1969), 912-914.

H. G. Dales

Department of Mathematics and Statistics

University of Lancaster

Lancaster LA1 4YF, United Kingdom

E-mail: g.dales@lancaster.ac.uk
A. Ülger

Department of Mathematics

Koç University

34450 Sariyer-Istanbul, Turkey

E-mail: aulger@ku.edu.tr

Received October 29, 2014

Revised version April 22, 2015 
